\title{
Relations in the Canonical Algebras on Surfaces.
}

\author{
KazUhiro Konno (*)
}

ABSTRACT - The degree bound for primitive generators and relations of the canonical ring of a minimal surface of general type are studied via Green's Koszul cohomology, assuming that the fixed part of the canonical linear system does not contain any Francia cycles. Slight refinements of the results due to Ciliberto and Reid are given.

\section{Introduction.}

Let $S$ be a non-singular, projective, minimal surface of general type defined over the complex number field $\mathrm{C}$. The canonical ring of $S$ is the graded C-algebra

$$
R\left(S, K_{S}\right)=\bigoplus_{m=0}^{+\infty} H^{0}\left(S, m K_{S}\right)
$$

This naive object has been studied by many authors in order to see what can be expected on the analogy of Max Noether's and Enriques-Petri's theorems for curves. We recall here some important results obtained so far. Ciliberto [4] showed, among other things, that $R\left(S, K_{S}\right)$ is generated in degrees $\leq 5$ under some reasonable conditions. Green [8] considered the case that the canonical linear system is free from base points in any dimension by applying his theory of Koszul cohomology groups (see also [9]). Reid [19] showed that it has the 1-2-3 property, that is, it is generated in degrees $\leq 3$ and related in degrees $\leq 6$, when $S$ is a regular surface with $K_{S}^{2} \geq 3$, $p_{g}(S) \geq 2$ which has an irreducible canonical curve on the canonical model.

(*) Indirizzo dell'A.: Department of Mathematics Graduate School of Science, Osaka University, Toyonaka, Osaka 560-0043, Japan.

E-mail: konno@math.sci.osaka-u.ac.jp

Partially supported by Grants-in-Aid for Scientific Research (B) (No. 16340008) by Japan Society for the Promotion of Science (JSPS). 
Mendes Lopes [17] considered surfaces with vanishing geometric genera and showed that $R\left(S, K_{S}\right)$ is generated in degrees $\leq 4$ provided that $2 K_{S}$ is free.

In this article, we study the degree bound for primitive homogeneous generators and relations of $R\left(S, K_{S}\right)$. The key point is the well-known result essentially due to Francia [7] and Reider [22] that $2 K_{S}$ is free except possibly when $p_{g}(S)=0$ and $K_{S}^{2} \leq 4$. We apply the machinery of Green's Koszul cohomology groups ([8], [9]) and show the following:

THEOREm A. Let $S$ be a minimal algebraic surface of general type such that $2 K_{S}$ is free. Then $R\left(S, K_{S}\right)$ is generated in degrees $\leq 5$ and related in degrees $\leq 10$. If furthermore $q(S)=0$, then $R\left(S, K_{S}\right)$ is generated in degrees $\leq 4$ and related in degrees $\leq 8$ except when $\left(p_{g}(S), K_{S}^{2}\right)=(2,1)$ and possibly $\left(p_{g}(S), K_{S}^{2}\right)=(0,3)$.

This is nothing more than what one naturally expects after Ciliberto's results on generators [4]. Recall that $R\left(S, K_{S}\right)$ needs a generator in degree 4 if the fixed part of $\left|K_{S}\right|$ contains a special configuration of curves called a Francia cycle, that is, an effective divisor $E$ with $p_{a}(E)=-E^{2}=1$ or $p_{a}(E)=2, E^{2}=0$, and many such surfaces are constructed in [4]. Hence the bound in Theorem A is sharp for regular surfaces in this sense.

On the other hand, it is an interesting problem [19] to study whether the canonical ring has the 1-2-3 property if the fixed part of $\left|K_{S}\right|$ does not contain any Francia cycles. We consider the problem for a regular surface whose canonical map is not composed of a pencil. However, partly because we do not know much about the fixed loci, we have to impose some extra restrictions modeled on fibred surfaces.

THEOREM B. Let $S$ be a minimal algebraic surface of general type with $p_{g}(S) \geq 2, q(S)=0$ and $K_{S}^{2} \geq 3$. Let $\left|K_{S}\right|=|M|+Z$ be the decomposition into its variable and fixed parts. Suppose that $|M|$ has an irreducible member. If one of the following conditions (1) and (2) is satisfied, then $R\left(S, K_{S}\right)$ is generated in degrees $\leq 3$ and related in $\leq 6$.

(1) $H^{1}\left(Z, \mathcal{O}_{Z}\right)=0$ (possibly $Z=0$ ).

(2) $Z$ does not contain a Francia cycle and decomposes as $Z=\Delta+\Gamma_{1}+\cdots+\Gamma_{n}$, where

(a) $\Delta$ is an effective divisor with $K_{S} \Delta=0$ (possibly $\Delta=0$ ), $\operatorname{Supp}(\Delta) \cap \operatorname{Supp}(Z-\Delta)=\emptyset$, and

(b) for each $i \in\{1, \ldots, n\}, \Gamma_{i}$ is a chain connected curve such that $K_{S} \Gamma_{i}>0, \mathcal{O}_{\Gamma_{i}}\left(-\Gamma_{i}\right)$ is nef, and $\mathcal{O}_{\Gamma_{i}}\left(\Gamma_{j}\right)$ is numerically trivial when $j \neq i$. 
If the fixed part supports at most exceptional sets of rational singular points, then we have $h^{1}\left(Z, \mathcal{O}_{Z}\right)=0$. Hence (1) can be regarded as a slight generalization of Reid's theorem [19] referred above. A curve $D$ is chain connected (cfr. [21]), if either it is irreducible, or for any proper subcurve $\Delta \preceq D$ there exists an irreducible component $A \preceq \Delta$ such that $(D-\Delta) A>0$. The restrictions in (2) are for technical reasons and should be replaced by a simpler assumption, e.g., the intersection form is negative semi-definite on $\operatorname{Supp}(Z)$. Note that, if a curve $\Gamma_{i}$ as in (2) exists, then the intersection form is negative semi-definite on $\operatorname{Supp}\left(\Gamma_{i}\right)$ and $\Gamma_{i}$ is nothing more than the $n u$ merical cycle on it in the terminology in [20]. The proof of Theorem B is based on the hyperplane section principle as in [19] and a result for chain connected curves similar to those in [15] and [13].

The organization of the paper is as follows. The first two sections are of preparatory nature. In $\S 1$, we recall and restate the fundamental tools, such as the duality theorem, the vanishing theorem and the $K_{p, 1}$ theorem, for Green's Koszul cohomology groups [9] in the form suitable for our purpose. We recall in $\S 2$ some important notions treating the connectedness of effective divisors and show Theorem 2.6 which is crucial for the proof of Theorem B, (2). In $\S 3$, we show Theorem A by studying the bicanonical maps. We need several existing results to manage some special cases. Especially when $p_{g}=q=0$, our proof heavily depends on [17]. Theorem B will be shown in $\S \S 4,5$. At the end of $\S 4$, we state a result for irregular surfaces similar to Theorem B, (1). In Appendix (\$6), we give a few remarks on the relative canonical algebras for fibrations and singularities, in order to supplement the results in [15] and [13] about Reid's 12-3 conjecture.

The author would like to thank the referee for his interest and stimulating suggestions.

\section{Koszul cohomology.}

In [8] and [9], Green developed the theory of Koszul cohomology groups which gives us a powerful tool for studying graded rings associated to line bundles. Although the results in [9] are stated for compact complex manifolds, it is not so hard to see that most of them hold also for singular objects if one makes suitable modifications. In this section, we restate three main results in [9] (the duality theorem [9, Theorem (2.c.6)], the vanishing theorem [9, Theorem (3.a.1)] and the $K_{p, 1}$ theorem [9, Theorem (3.c.1)]) in the forms suitable for our purpose. 
Let $X$ be a projective scheme (over $\mathrm{C}$ ) of pure dimension $n$. Let $\mathcal{L}$ is an invertible sheaf on $X$ and take a subspace $W \subseteq H^{0}(X, \mathcal{L})$ giving us a linear system which is free from base points. Then $W$ defines a morphism $\Phi_{W}: X \rightarrow \mathbb{P}(W)$. By pulling back the exact sequence $0 \rightarrow \Omega_{\mathrm{P}(W)}^{1}(-1) \rightarrow$ $W \otimes_{\mathrm{C}} \mathcal{O}_{\mathrm{P}(W)} \rightarrow \mathcal{O}_{\mathrm{P}(W)}(1) \rightarrow 0$, one obtains $0 \rightarrow \mathcal{E} \rightarrow W \otimes_{\mathbb{C}} \mathcal{O}_{X} \rightarrow \mathcal{L} \rightarrow 0$, which gives us

$$
0 \rightarrow \bigwedge^{p} \mathcal{E} \rightarrow \bigwedge^{p} W \otimes \mathcal{O}_{X} \rightarrow \bigwedge^{p-1} \mathcal{E} \otimes \mathcal{L} \rightarrow 0
$$

for any positive integer $p$. In particular, $\bigwedge^{r} \mathcal{E} \simeq \mathcal{L}^{\vee}$ if $r=\operatorname{dim} W-1=$ $=\operatorname{rk}(\mathcal{E})$. Let $\mathcal{M}$ be an invertible sheaf on $X$. Then tensoring $\mathcal{M} \otimes \mathcal{L}^{\otimes q}$ $(q \in Z)$ to the above, one gets

$(S e q)_{p, q}: \quad 0 \rightarrow \mathcal{E}_{p, q}(\mathcal{M}) \rightarrow \bigwedge^{p} W \otimes \mathcal{M} \otimes \mathcal{L}^{\otimes q} \rightarrow \mathcal{E}_{p-1, q+1}(\mathcal{M}) \rightarrow 0$,

where $\mathcal{E}_{p, q}(\mathcal{M})=\bigwedge^{p} \mathcal{E} \otimes \mathcal{M} \otimes \mathcal{L}^{\otimes q}$. Let

$$
d_{p, q}: \bigwedge^{p} W \otimes H^{0}\left(X, \mathcal{M} \otimes \mathcal{L}^{\otimes q}\right) \rightarrow \bigwedge^{p-1} W \otimes H^{0}\left(X, \mathcal{M} \otimes \mathcal{L}^{\otimes(q+1)}\right)
$$

be the composite of $\bigwedge^{p} W \otimes H^{0}\left(X, \mathcal{M} \otimes \mathcal{L}^{\otimes q}\right) \rightarrow H^{0}\left(X, \mathcal{E}_{p-1, q+1}(\mathcal{M})\right)$ and the natural injection $H^{0}\left(X, \mathcal{E}_{p-1, q+1}(\mathcal{M})\right) \hookrightarrow \bigwedge^{p-1} W \otimes H^{0}\left(X, \mathcal{M} \otimes \mathcal{L}^{\otimes(q+1)}\right)$ obtained from $(S e q)_{p, q}$ and $(S e q)_{p-1, q+1}$, respectively. Then $d_{p, q} \circ d_{p+1, q-1}=0$. The Koszul cohomology group is nothing but

$$
\begin{aligned}
K_{p, q}(X, \mathcal{M}, \mathcal{L}, W) & =\operatorname{Ker}\left(d_{p, q}\right) / \operatorname{Im}\left(d_{p+1, q-1}\right) \\
& \simeq \operatorname{Coker}\left\{\bigwedge^{p+1} W \otimes H^{0}\left(X, \mathcal{M} \otimes \mathcal{L}^{\otimes(q-1)}\right) \rightarrow H^{0}\left(X, \mathcal{E}_{p, q}(\mathcal{M})\right)\right\} .
\end{aligned}
$$

Theorem 1.1 (Duality Theorem [9, Theorem (2.c.6)]). Let $X$ be a Gorenstein projective scheme of pure dimension $n$ and $\mathcal{L}, \mathcal{M}$ invertible sheaves on $X$. If $W \subseteq H^{0}(X, \mathcal{L})$ denotes a subspace of dimension $r+1$ inducing a linear system which is free from base points, then $K_{p, q}(X, \mathcal{M}, \mathcal{L}, W)$ is dual to $K_{r-n-p, n+1-q}\left(X, \omega_{X} \otimes \mathcal{M}^{\vee}, \mathcal{L}, W\right)$ provided that $H^{i}\left(X, \mathcal{M} \otimes \mathcal{L}^{\otimes(q-i)}\right)=H^{i}\left(X, \mathcal{M} \otimes \mathcal{L}^{\otimes(q-i-1)}\right)=0$ for $1 \leq i \leq n-1$, where $\omega_{X}$ denotes the dualizing sheaf.

Proof. We have a natural injection $K_{p, q}(X, \mathcal{M}, \mathcal{L}, W) \hookrightarrow$ $\hookrightarrow H^{1}\left(X, \mathcal{E}_{p+1, q-1}(\mathcal{M})\right)$. Consider the coboundary map

$$
\delta_{i}: H^{i}\left(X, \mathcal{E}_{p+i, q-i}(\mathcal{M})\right) \rightarrow H^{i+1}\left(X, \mathcal{E}_{p+i+1, q-i-1}(\mathcal{M})\right)
$$


obtained from $(S e q)_{p+i+1, q-i-1}$ for $1 \leq i \leq n-1$. If $H^{i}\left(X, \mathcal{M} \otimes \mathcal{L}^{\otimes(q-i-1)}\right)=0$ for $1 \leq i \leq n-1$, then $\delta_{i}$ is injective for $1 \leq i \leq n-1$. Therefore, $K_{p, q}(X, \mathcal{M}, \mathcal{L}, W) \hookrightarrow H^{n-1}\left(X, \mathcal{E}_{p+n-1, q-n+1}(\mathcal{M})\right)$ and

$$
\begin{aligned}
0 \rightarrow H^{n-1}\left(X, \mathcal{E}_{p+n-1, q-n+1}(\mathcal{M})\right) \rightarrow H^{n}\left(X, \mathcal{E}_{p+n, q-n}(\mathcal{M})\right) & \\
& \rightarrow \bigwedge^{p+n} W \otimes H^{n}\left(X, \mathcal{M} \otimes \mathcal{L}^{\otimes(q-n)}\right)
\end{aligned}
$$

is exact. By the Serre duality theorem, the dual sequence of the latter is

$$
\begin{aligned}
\bigwedge^{p+n} W^{\vee} \otimes H^{0}\left(X, \omega_{X} \otimes \mathcal{M}^{\vee} \otimes \mathcal{L}^{\otimes(n-q)}\right) & \rightarrow H^{0}\left(X, \omega_{X} \otimes \mathcal{E}_{p+n, q-n}(\mathcal{M})^{\vee}\right) \\
& \rightarrow H^{1}\left(X, \omega_{X} \otimes \mathcal{E}_{p+n-1, q-n+1}(\mathcal{M})^{\vee}\right) \rightarrow 0 .
\end{aligned}
$$

Since we have $\bigwedge^{p+n} W^{\vee} \simeq \bigwedge^{r+1-p-n} W$ and

$$
\mathcal{E}_{p+n, q-n}(\mathcal{M})^{\vee} \simeq \bigwedge^{p+n} \mathcal{E}^{\vee} \otimes \mathcal{M}^{\vee} \otimes \mathcal{L}^{\otimes(n-q)} \simeq \bigwedge^{r-n-p} \mathcal{E} \otimes \mathcal{M}^{\vee} \otimes \mathcal{L}^{\otimes(n-q+1)}
$$

by $\bigwedge^{r+1} W \simeq \mathrm{C}$ and $\bigwedge^{r} \mathcal{E} \simeq \mathcal{L}^{\vee}$, respectively, the above exact sequence is nothing more than

$$
\begin{array}{r}
\bigwedge^{r+1-p-n} W \otimes H^{0}\left(X, \omega_{X} \otimes \mathcal{M}^{\vee} \otimes \mathcal{L}^{\otimes(n-q)}\right) \rightarrow H^{0}\left(X, \mathcal{E}_{r-n-p, n-q+1}\left(\omega_{X} \otimes \mathcal{M}^{\vee}\right)\right) \\
\rightarrow H^{1}\left(X, \mathcal{E}_{r-n-p+1, n-q}\left(\omega_{X} \otimes \mathcal{M}^{\vee}\right)\right) \rightarrow 0
\end{array}
$$

This shows that the kernel of $H^{n}\left(X, \mathcal{E}_{p+n, q-n}(\mathcal{M})\right) \rightarrow \bigwedge^{p+n} W \otimes$ $\otimes H^{n}\left(X, \mathcal{M} \otimes \mathcal{L}^{\otimes(q-n)}\right)$ is the dual space of $K_{r-n-p, n+1-q}\left(X, \omega_{X} \otimes \mathcal{M}^{\vee}, \mathcal{L}, W\right)$. Hence $K_{p, q}(X, \mathcal{M}, \mathcal{L}, W)$ is mapped injectively to $K_{r-n-p, n+1-q}\left(X, \omega_{X} \otimes\right.$ $\left.\otimes \mathcal{M}^{\vee}, \mathcal{L}, W\right)^{\vee}$. Similarly, if $H^{i}\left(X, \mathcal{M} \otimes \mathcal{L}^{\otimes(q-i)}\right)=0$ for $1 \leq i \leq n-1$, then $K_{r-n-p, n+1-q}\left(X, \omega_{X} \otimes \mathcal{M}^{\vee}, \mathcal{L}, W\right) \rightarrow K_{p, q}(X, \mathcal{M}, \mathcal{L}, W)^{\vee}$ is injective, since $H^{j}\left(X, \omega_{X} \otimes \mathcal{M}^{\vee} \otimes \mathcal{L}^{\otimes(n-q-j)}\right)$ is dual to $H^{n-j}\left(X, \mathcal{M} \otimes \mathcal{L}^{\otimes(q-(n-j))}\right)$ by the Serre duality theorem.

We remark that the duality theorem holds for Gorenstein curves without additional cohomological conditions. See also [13, Lemma 1.2.1] for curves on a smooth surface. The following two theorems can be shown as in [9] without major changes. Hence we only give the statements without proofs.

Theorem 1.2 (Vanishing Theorem [9, Theorem (3.a.1)]). Let $X$ be an irreducible projective variety. If $\mathcal{L}, \mathcal{M}$ are invertible sheaves on $X$ and $W \subseteq H^{0}(X, \mathcal{L})$ is a subspace, then $K_{p, q}(X, \mathcal{M}, \mathcal{L}, W)=0$ provided that $h^{0}\left(X, \mathcal{M} \otimes \mathcal{L}^{\otimes q}\right) \leq p$. 
For simplicity, we write $K_{p, q}(X, \mathcal{L})$ instead of $K_{p, q}\left(X, \mathcal{L}, \mathcal{L}, H^{0}(X, \mathcal{L})\right)$.

Theorem 1.3 (The $K_{p, 1}$ Theorem [9, Theorem (3.c.1)]). Let $X$ be an irreducible projective variety, $\mathcal{L}$ an invertible sheaf on $X$ with $h^{0}(X, \mathcal{L})=$ $=r+1$ and put $m=\operatorname{dim} \Phi_{\mathcal{L}}(X)$. Then the following hold.

(1) $K_{p, 1}(X, \mathcal{L})=0$ for $p>r-m$.

(2) $K_{r-m, 1}(X, \mathcal{L})=0$ unless $\Phi_{\mathcal{L}}(X)$ is an m-fold of minimal degree.

(3) $K_{r-m-1,1}(X, \mathcal{L})=0$ unless either $\operatorname{deg} \Phi_{\mathcal{L}}(X) \leq r+2-m$ or $\Phi_{\mathcal{L}}(X)$ lies on an $(m+1)$-fold of minimal degree.

The irreducibility of $X$ is not essential in the vanishing theorem, while it is an important assumption in the $K_{p, 1}$ theorem, since we require the uniform position property for the zero cycle obtained as a general linear section of $\Phi_{\mathcal{L}}(X)$. See [8] and [9] for the detail.

We recall one more lemma from [8] which will be used frequently.

LEmma 1.4 ([8]). Let $\mathcal{L}$ be an invertible sheaf on a projective scheme $X$ and let $k$ be the smallest positive integer such that $\mathcal{L}^{\otimes k}$ is generated by global sections. Assume that the graded ring $R(X, \mathcal{L})=\bigoplus_{m>0} H^{0}\left(X, \mathcal{L}^{\otimes m}\right)$ is generated in degrees $\leq d$. Put

$$
d_{0}=\min _{n \in Z_{\geq 0}}\left\{H^{0}\left(X, L^{\otimes k}\right) \otimes H^{0}\left(X, L^{\otimes(m-k)}\right) \rightarrow H^{0}\left(X, L^{\otimes m}\right)\right. \text { is surjec- }
$$
tive for any $m>n$ \}

$$
\begin{array}{r}
d_{1} \min _{n \in Z_{\geq 0}}\left\{\bigwedge^{2} H^{0}\left(X, L^{\otimes k}\right) \otimes H^{0}\left(X, L^{\otimes(m-2 k)}\right) \rightarrow H^{0}\left(X, L^{\otimes k}\right) \otimes H^{0}\left(X, L^{\otimes(m-k)}\right)\right. \\
\left.\rightarrow H^{0}\left(X, L^{\otimes m}\right) \text { is exact at the middle term for any } m<n\right\}
\end{array}
$$

Then $R(X, \mathcal{L})$ is related in degrees $\leq \max \left\{d+d_{0}, d_{1}\right\}$. If $d_{0}$ is not a multiple of $d$, then $R(X, \mathcal{L})$ is related in degrees $\leq \max \left\{d+d_{0}-1, d_{1}\right\}$.

Proof. Though this is contained in the proof of [8, Theorem 3.11], we recall the argument for the later use.

Choosing a minimal set of homogeneous generators of $R(X, \mathcal{L})$, we have a surjective homomorphism $\Phi: \Omega \rightarrow R(X, \mathcal{L})$, where $\Omega$ is a polynomial ring over C. We let $\Omega=\bigoplus_{m>0} \Omega_{m}$ be the decomposition into the graded pieces such that $\Phi$ becomes a homomorphism of graded algebras. The kernel $I$ of $\Phi$ is a homogeneous ideal and we denote it as $I=\bigoplus_{m \geq 0} I_{m}$. We let $\left\{\xi_{1}, \ldots, \xi_{N}\right\}$ be a basis for $\Omega_{k}$. 
Assume that $n>\max \left\{d+d_{0}, d_{1}\right\}$ and let $F \in I_{n}$ be a relation in degree $n$. We shall show that $F \equiv 0$ modulo relations in lower degrees. Let $G$ be any monomial appearing in $F$. Since $n>d+d_{0}$, we see that $G$ can be divided by a monomial $G_{1}$ satisfying $d_{0}<\operatorname{deg} G_{1} \leq d+d_{0}$. Then, since $\operatorname{deg} G_{1}>d_{0}, \Phi\left(G_{1}\right)$ can be expressed as a sum of products of degree $k$ and degree $n-k$ elements in $R(X, \mathcal{L})$. Therefore, we can write $F=\sum_{i=1}^{N} \xi_{i} F_{i}$ modulo relations in degrees $\leq \max \left\{n-k, d+d_{0}\right\}$, where the $F_{i}$ 's are homogeneous polynomials of degree $n-k$. Then, since $n>d_{1}$, we can find polynomials $F_{i j}$ of degree $n-2 k$ satisfying $F_{i}=\sum \xi_{j} F_{i j}$ and $F_{i j}=-F_{j i}$, modulo relations in degrees $<n$. Then we obtain $F=\sum_{i} \xi_{i} F_{i}=$ $=\sum_{i \leq j} \xi_{i} \xi_{j}\left(F_{i j}+F_{j i}\right)=0$ modulo relations in lower degrees.

If $d_{0}$ is not a multiple of $d$, then the above argument works also when $n=d_{0}+d$.

\section{Chain connected curves.}

A non-zero effective divisor on a non-singular surface will be simply called a curve. In this section, we recall basic notions about the connectedness of curves from [5, Appendix] and [21] and collect some results for the later use. See also [20]. In some sense, this section is a reconsideration of [21].

Let $D$ be a curve. For an integer $k, D$ is numerically $k$-connected if $D_{1} D_{2} \geq k$ holds for any decomposition $D=D_{1}+D_{2}$ where $D_{1}, D_{2}$ are curves. Note that a nef and big curve is necessarily 1-connected. In particular, so is a canonical curve of a minimal surface of general type.

Definition 2.1. A numerically 2-connected curve $E$ is called a Francia cycle, if either (i) $p_{a}(E)=1$ and $E^{2}=-1$, or (ii) $p_{a}(E)=2$ and $E^{2}=0$.

A curve as in (i) will be sometimes called a ( -1$)$ elliptic cycle.

If $\operatorname{Supp}(D)$ is connected and the intersection form is negative semidefinite on it, then $D$ is called the numerical cycle if $D$ is the smallest curve such that $-D$ is nef on its support. When the intersection form is negative definite, that is, $\operatorname{Supp}(D)$ is the exceptional set of a normal surface singularity, the numerical cycle is usually called the fundamental cycle. A curve $D$ is chain connected ([21]), if either it is irreducible, or for any proper subcurve $\Delta \preceq D$ there exists an irreducible component $A \preceq \Delta$ such that $(D-\Delta) A>0$. It is easy to see that numerical cycles and 1-connected curves are chain connected. 
LEMma 2.2. For a chain connected curve D, the following hold.

(1) $h^{0}\left(D, \mathcal{O}_{D}\right)=1$ and a non-zero element of $H^{0}\left(D, \mathcal{O}_{D}\right)$ does not vanish identically on any component.

(2) If $L$ is a nef line bundle on $D$, then $H^{0}(D,-L) \neq 0$ if and only if $\mathcal{O}_{D}(L) \simeq \mathcal{O}_{D}$

Proof. We first show (2) by using an argument in [5, Appendix]. Let $s \in H^{0}(D,-L)$ be a non-zero element. If $s$ vanishes identically on some components of $D$, we let $\Delta$ be the biggest subcurve of $D$ on which $s$ vanishes identically. Then $s$ induces a non-zero element of $H^{0}(D-\Delta,-L-\Delta)$ which does not vanish identically on any component of $D-\Delta$. This implies that $-L-\Delta$ is nef on $D-\Delta$. On the other hand, by the chain connectedness of $D$, there is an irreducible component $A \preceq D-\Delta$ such that $\Delta A>0$. Then $\operatorname{deg}(-L-\Delta)_{\mid A} \leq-\Delta A<0$, which contradicts what we have just seen above. Hence $s$ does not vanish identically on any component of $D$. Then $-L$ is numerically trivial, since $L$ is nef, and the nowhere vanishing section $s$ induces an isomorphism $\mathcal{O}_{D} \simeq \mathcal{O}_{D}(-L)$.

To show (1), we assume that $h^{0}\left(D, \mathcal{O}_{D}\right)>1$. Then we can find a non-zero element $s \in H^{0}\left(D, \mathcal{O}_{D}\right)$ which vanishes identically on some components of $D$. Take the biggest subcurve $\Delta$ on which $s$ vanishes identically and copy the first half of the proof of (2) with $-L$ being replaced by $\mathcal{O}_{D}$. Then it will immediately lead us to a contradiction. Hence $h^{0}\left(D, \mathcal{O}_{D}\right)=1$. The last assertion in (1) is clear from the above proof of (2).

Here we recall the following easy lemma whose proof can be found in [13, Lemma 2.2.1].

Lemma 2.3. Let $D$ be a curve and $L$ a line bundle on D. Take an irreducible component $A \preceq D$ and let $\Delta$ be a minimal curve containing $A$ such that the restriction map $H^{0}(D, L) \rightarrow H^{0}(\Delta, L)$ is surjective. Then one of the following holds.

(1) $K_{\Delta}-L$ is nef on $\Delta$.

(2) $A$ is of multiplicity one in $\Delta$ and $K_{\Delta}-L$ is nef on $\Delta-A$. Furthermore, $\left.\operatorname{deg}(L-(\Delta-A))\right|_{A}>\operatorname{deg} K_{A}$ and the image of the restriction map $H^{0}(D, L) \rightarrow H^{0}(A, L)$ contains that of the natural inclusion $H^{0}(A, L-(\Delta-A)) \rightarrow H^{0}(A, L)$.

If $|L|$ has no base points on $A \cap(\Delta-A)$ in (2), then $h^{0}(A, L) \geq$ $\geq h^{0}(A, L-(\Delta-A))+1$. 
Proposition 2.4. Let $D$ be chain connected and $L$ a line bundle on $D$ such that $L-K_{D}$ is nef and $\operatorname{deg}\left(L-K_{D}\right)>0$. If $x \in \operatorname{Bs}|L|$, then there exists a subcurve $\Delta$ of $D$ satisfying the following properties.

(1) $x$ is a non-singular point of $\Delta$.

(2) The restriction map $H^{0}(D, L) \rightarrow H^{0}(\Delta, L)$ is surjective.

(3) $\mathcal{O}_{A}(L) \simeq \omega_{\Delta} \otimes \mathcal{O}_{A}(x)$, where $A$ denotes the unique irreducible component of $\Delta$ through $x$.

(4) $K_{\Delta}-L$ is nef on $\Delta-A$.

Proof. We may assume that $D$ is reducible. Take an irreducible component $A$ through $x$ and let $\Delta=\Delta_{A}$ be a minimal subcurve of $D$ such that $A \preceq \Delta$ and $H^{0}(D, L) \rightarrow H^{0}(\Delta, L)$ is surjective.

We first assume that $\Delta=A$. We have $\left.\operatorname{deg} L\right|_{A}=\left.\operatorname{deg}\left(L-K_{D}\right)\right|_{A}+$ $+\operatorname{deg} K_{A}+(D-A) A$. Since $L-K_{D}$ is nef and $D$ is chain connected, we get $\left.\operatorname{deg} L\right|_{A}>\operatorname{deg} K_{A}$. Since $x \in \operatorname{Bs}\left|\mathcal{O}_{A}(L)\right|, x$ is a non-singular point of $A$ and $\mathcal{O}_{A}(L) \simeq \omega_{A}(x)$.

We next assume that $\Delta=D$. Since $L-K_{D}$ is nef and $\operatorname{deg}\left(L-K_{D}\right)>0$, it follows from Lemma 2.3 that $A$ is of multiplicity one in $D, K_{D}-L$ is nef on $D-A$ and $\left.\operatorname{deg}(L-(D-A))\right|_{A}>\operatorname{deg} K_{A}$. Note that $H^{0}(D, L) \rightarrow$ $\rightarrow H^{0}(D-A, L)$ is surjective, because $H^{1}(A, L-(D-A))=0$. Hence, we can assume that $x \notin A \cap(D-A)$, because, otherwise, there exists another irreducible component $B$ through $x$ and then $\Delta_{B} \preceq D-A$ by what we have just seen which enables us to argue with $\left(B, \Delta_{B}\right)$ instead of $\left(A, \Delta_{A}\right)$. Then $x \in \operatorname{Bs}\left|\mathcal{O}_{A}(L-(D-A))\right|$. Since $\left.\operatorname{deg}(L-(D-A))\right|_{A}>\operatorname{deg} K_{A}, x$ has to be a non-singular point of $A$ and we see that $\mathcal{O}_{A}(L-(D-A)) \simeq \omega_{A}(x)$ which is equivalent to $\mathcal{O}_{A}(L) \simeq \omega_{D} \otimes \mathcal{O}_{A}(x)$. Note that $x$ is also a nonsingular point of $D$ by $x \notin A \cap(D-A)$.

We finally assume that $\Delta \neq A$ and $\Delta \neq D$. We have $K_{\Delta}-L=$ $=K_{D}-L-(D-\Delta)$ on $\Delta$. Since $D$ is chain connected, we can find an irreducible component $B \preceq \Delta$ such that $(D-\Delta) B>0$. For such $B$, we get $\left.\operatorname{deg}\left(K_{\Delta}-L\right)\right|_{B}<0$. Hence $K_{\Delta}-L$ is not nef on $\Delta$ and we are in the situation of Lemma 2.3, (2). Since $H^{0}(\Delta, L) \rightarrow H^{0}(\Delta-A, L)$ is surjective, we may assume that $x \notin A \cap(\Delta-A)$ as in the previous case. Then we can conclude that $x$ is a non-singular point of $A$ and $\mathcal{O}_{A}(L-(\Delta-A)) \simeq \omega_{A}(x)$. This shows $\mathcal{O}_{A}(L) \simeq \omega_{\Delta} \otimes \mathcal{O}_{A}(x)$.

COROLLARY 2.5. Let D be a chain connected curve on a smooth surface $S$ such that $K_{S}$ and $-D$ are both nef on $D$ and $D^{2}<0$. If $L$ is a line bundle on $D$ such that $L-r K_{S}$ is a nef $\mathrm{Q}$-bundle for some rational number $r>1$, then $H^{1}(D, L)=0$ and $|L|$ has no base points. 
Proof. We may assume that $D$ is reducible. We have $L-K_{D}=$ $=L-r K_{S}+(r-1) K_{S}-D$ on $D$. Hence $L-K_{D}$ is nef of positive degree. By the Serre duality theorem, $H^{1}(D, L)^{\vee} \simeq H^{0}\left(D, K_{D}-L\right)$. So we get $H^{1}(D, L)=0$, because $D$ is chain connected.

We show that $|L|$ is free from base points. We first remark that the intersection form is negative definite on $\operatorname{Supp}(D)$ and $D$ is the numerical cycle, since $\operatorname{Supp}(D)$ is connected, $\mathcal{O}_{D}(-D)$ is nef and $D^{2}<0$. Suppose now that $x$ is a base point of $|L|$. Then there exists a subcurve $\Delta$ and an irreducible component $A$ as in Proposition 2.4. We have $\mathcal{O}_{\Delta}\left(K_{\Delta}-L\right)=\mathcal{O}_{\Delta}\left(r K_{S}-L-(r-1) K_{S}+\Delta\right)$. Since $\mathcal{O}_{A}\left(L-K_{\Delta}\right) \simeq \mathcal{O}_{A}(x)$, we have $\Delta A=(r-1) K_{S} A+\left(L-r K_{S}\right) A-1$. Since $K_{\Delta}-L$ is nef on $\Delta-A$, we have $\Delta(\Delta-A) \geq\left(L-r K_{S}\right)(\Delta-A)+(r-1) K_{S}(\Delta-A)$. In sum, we get $\Delta^{2}=\Delta A+\Delta(\Delta-A) \geq\left(L-r K_{S}\right) \Delta+(r-1) K_{S} \Delta-1$. If $\Delta=D$ or $\Delta=A$, then, since $\Delta^{2}<0$, we must have $\Delta^{2}=-1$ and $K_{S} \Delta=0$, which is impossible for the reason of parity. Assume that $\Delta \neq D$ and $\Delta \neq A$. Since $D$ is chain connected, we can find an irreducible component $B$ of $\Delta$ satisfying $(D-\Delta) B>0$. Then $0 \geq D B>\Delta B$, which shows that $K_{\Delta}-L$ is of negative degree on $B$. Hence we get $B=A$. Now, it follows from $0>\Delta A==\left(L-r K_{S}\right) A+(r-1) K_{S} A-1$ that $\left.\operatorname{deg} L\right|_{A}=K_{S} A=0$ and $-1=\Delta A==(\Delta-A) A+A^{2}$. The last shows that $A^{2}=-1$ and $(\Delta-A) A=0$, because $A^{2}<0$ and $A$ is of multiplicity one in $\Delta$. It is again impossible for the reason of parity.

Let $D$ be a chain connected curve on a non-singular surface $S$. Take an arbitrary subcurve $A_{1}$ of $D$ and put $D_{1}=A_{1}$. If $D_{1} \neq D$, then there is an irreducible component $A_{2}$ of $D-D_{1}$ such that $D_{1} A_{2}>0$. We put $D_{2}=D_{1}+A_{2}$. If $D_{i}$ is defined and $D_{i} \neq D$, then we take an irreducible component $A_{i+1}$ of $D-D_{i}$ such that $D_{i} A_{i+1}>0$, and put $D_{i+1}=D_{i}+A_{i+1}$. In this way, we get a composition series $\left\{A_{i}\right\}_{i=1}^{N}$ such that $D=\sum_{i=1}^{N} A_{i}$. It is said to be irreducible, if the first curve $A_{1}$ is also irreducible. We put $X_{i}=D-D_{N-i}$ for $0 \leq i \leq N$.

THEOREM 2.6. Let $D$ be a chain connected curve on a non-singular surface $S$ with $K_{S}^{2}>0$ such that $K_{S}$ and $-D$ are both nef on $D$. For $\alpha=1,2$, let $L_{\alpha}$ be a line bundle on $D$ such that $L_{\alpha}-2 K_{S}$ is nef. Then the multiplication map

$$
H^{0}\left(D, L_{1}\right) \otimes H^{0}\left(D, L_{2}\right) \rightarrow H^{0}\left(D, L_{1}+L_{2}\right)
$$

is surjective, unless there exists a Francia cycle $E \preceq D$ on which $L_{1}$ and $L_{2}$ are both numerically equivalent to $2 K_{S}$. 
Proof. Since $D$ is chain connected and $\mathcal{O}_{D}(-D)$ is nef, the intersection form is negative semi-definite on $\operatorname{Supp}(D)$ and $D$ is nothing more than the numerical cycle on its support. Furthermore, for a subcurve $\Delta \preceq D$ with $K_{S} \Delta=0$, we have $\Delta^{2}<0$ by Hodge's index theorem, since $K_{S}^{2}>0$.

If $D$ is irreducible, it is easy to check that the multiplication map is surjective unless $D$ itself is a Francia cycle and $L_{1} \equiv L_{2} \equiv 2 K_{S}$ on $D$, where the symbol $\equiv$ means the numerical equivalence. So we may assume that $D$ is reducible.

We first consider the extremal case that $D^{2}=0$. Then $\mathcal{O}_{D}(D)$ is numerically trivial and $D$ is 1-connected. We have $K_{D}=\left.\left(K_{S}+D\right)\right|_{D}$ which is nef in the present case. Hence $p_{a}(D) \geq 1$. If $p_{a}(D)=1$, then $K_{S} D=D^{2}=0$, which is impossible by Hodge's index theorem because $K_{S}^{2}>0$. Hence we have $p_{a}(D) \geq 2$. Since $L_{\alpha}-2 K_{D}=\left(L_{\alpha}-2 K_{S}\right)-\left.2 D\right|_{D}$ is nef, the assertion follows from [13, Theorem I].

We assume that $D^{2}<0$. Then the intersection form is negative definite on $\operatorname{Supp}(D)$. We follow Laufer's argument [15]. We take an irreducible composition series $\left\{A_{i}\right\}_{i=1}^{N}$ for $D$ satisfying $D A_{1}<0$. Since $L_{\alpha}-2 K_{S}$ and $-D$ are nef on $D$, we can show that $H^{1}\left(D, L_{\alpha}\right)=0$ and the restriction map $H^{0}\left(X_{N-i+1}, L_{\alpha}\right) \rightarrow H^{0}\left(X_{N-i}, L_{\alpha}\right)$ is surjective. This in particular implies that $H^{0}\left(D, L_{\alpha}\right) \rightarrow H^{0}\left(X_{k}, L_{\alpha}\right)$ is surjective for $0<k<N, \alpha=1,2$. Furthermore, $\left|L_{\alpha}\right|$ is free from base points by Corollary 2.5. We have the exact sequence

$$
0 \rightarrow H^{0}\left(A_{i}, L-X_{N-i}\right) \rightarrow H^{0}\left(X_{N-i+1}, L\right) \rightarrow H^{0}\left(X_{N-i}, L\right) \rightarrow 0
$$

for $\quad L=L_{1}, \quad L_{2}, \quad L_{1}+L_{2}$. Then we see that $H^{0}\left(X_{N-i+1}, L_{1}\right) \otimes$ $\otimes H^{0}\left(X_{N-i+1}, L_{2}\right) \rightarrow H^{0}\left(X_{N-i+1}, L_{1}+L_{2}\right)$ is surjective provided that so are $H^{0}\left(X_{N-i}, L_{1}\right) \otimes H^{0}\left(X_{N-i}, L_{2}\right) \rightarrow H^{0}\left(X_{N-i}, L_{1}+L_{2}\right)$ and

$$
\begin{array}{r}
H^{0}\left(A_{i}, L_{1}-X_{N-i}\right) \otimes W_{2, i} \oplus W_{1, i} \otimes H^{0}\left(A_{i}, L_{2}-X_{N-i}\right) \\
\rightarrow H^{0}\left(A_{i}, L_{1}+L_{2}-X_{N-i}\right),
\end{array}
$$

where $W_{\alpha, i}=\operatorname{Im}\left\{H^{0}\left(D, L_{\alpha}\right) \rightarrow H^{0}\left(A_{i}, L_{\alpha}\right)\right\}$. By induction, in order to show that $H^{0}\left(D, L_{1}\right) \otimes H^{0}\left(D, L_{2}\right) \rightarrow H^{0}\left(D, L_{1}+L_{2}\right)$ is surjective, it suffices to show that (2.1) is surjective for any $i, 1 \leq i \leq N$. Since $L_{\alpha}-2 K_{S}$ is nef, we have $\left.\operatorname{deg}\left(L_{\alpha}-K_{S}\right)\right|_{A_{i}} \geq\left.\operatorname{deg} K_{S}\right|_{A_{i}} \geq 0$. If $L_{\alpha}-K_{S}$ is of degree zero on $A_{i}$, then $A_{i}$ is a ( - 2)-curve and (2.1) is clearly surjective, because $\left.L_{\alpha}\right|_{A_{i}}$ is trivial and $\operatorname{Bs}\left|L_{\alpha}\right|=\emptyset$. This enables us to assume that $\left.\operatorname{deg}\left(L_{\alpha}-K_{S}\right)\right|_{A_{i}}>0$ for $\alpha=1,2$ in what follows. We denote by $\Delta_{\alpha, i}$ a minimal subcurve of $D$ containing $A_{i}$ such that $H^{0}\left(D, L_{\alpha}\right) \rightarrow H^{0}\left(\Delta_{\alpha, i}, L_{\alpha}\right)$ is surjective.

We assume that $\left.\operatorname{deg} L_{1}\right|_{A_{i}} \geq\left.\operatorname{deg} L_{2}\right|_{A_{i}}$ and consider the multiplication map $\mu_{2, i}: H^{0}\left(A_{i}, L_{1}-X_{N-i}\right) \otimes W_{2, i} \rightarrow H^{0}\left(A_{i}, L_{1}+L_{2}-X_{N-i}\right)$. We shall 
show that either $\mu_{2, i}$ is surjective or there is a Francia cycle $\Delta$ containing $A_{i}$ and $L_{2} \equiv 2 K_{S}$ on $\Delta$. By Theorems 1.1 and $1.2, \mu_{2, i}$ is surjective if $h^{0}\left(A_{i}, K_{A_{i}}+L_{2}-L_{1}+X_{N-i}\right) \leq \operatorname{dim} W_{2, i}-2$. Hence we only have to check that

$$
\operatorname{dim} W_{2, i} \geq\left\{\begin{array}{l}
p_{a}\left(A_{i}\right)+2, \quad \text { if } K_{A_{i}}+L_{2}-L_{1}+X_{N-i} \text { is special, } \\
p_{a}\left(A_{i}\right)+1+\left.\operatorname{deg}\left(L_{2}-L_{1}\right)\right|_{A_{i}}+\left(D-D_{i-1}\right) A_{i}-A_{i}^{2} \\
\text { otherwise }
\end{array}\right.
$$

Assume that $W_{2, i}=H^{0}\left(A_{i}, L_{2}\right)$. Then $\operatorname{dim} W_{2, i}=\left.\operatorname{deg} L_{2}\right|_{A_{i}}+1-p_{a}\left(A_{i}\right)$. The inequality $\operatorname{dim} W_{2, i} \geq p_{a}\left(A_{i}\right)+2$ is equivalent to $\left.\operatorname{deg}\left(L_{2}-K_{S}\right)\right|_{A_{i}}-$ $-A_{i}^{2} \geq 3$, which fails only when $K_{S} A_{i}=1, A_{i}^{2}=-1$ and $\left.\operatorname{deg} L_{2}\right|_{A_{i}}=2$, that is, $A_{i}$ is a Francia cycle of arithmetic genus one on which $L_{2} \equiv 2 K_{S}$. The second inequality in (2.2) is equivalent to $\left.\operatorname{deg}\left(L_{1}-K_{S}\right)\right|_{A_{i}}-D A_{i}+$ $+D_{i-1} A_{i} \geq 2$, which always holds under our assumptions.

Assume that $W_{2, i} \neq H^{0}\left(A_{i}, L_{2}\right)$ and put $\Delta_{i}:=\Delta_{2, i}$. Since $H^{0}\left(D, L_{2}\right) \rightarrow$ $\rightarrow H^{0}\left(X_{N-i+1}, L_{2}\right)$ is surjective, we have $\Delta_{i} \preceq X_{N-i+1}$. In particular, we have $\Delta_{i}=D$ only when $i=1$. It follows from Lemma 2.3 that we have either (i) $\Delta_{i}-\left(L_{2}-K_{S}\right)$ is nef on $\Delta_{i}$, or (ii) $A_{i}$ is of multiplicity one in $\Delta_{i}$, $\Delta_{i}-\left(L_{2}-K_{S}\right)$ is nef on $\Delta_{i}-A_{i}$ and $\mathcal{O}_{A_{i}}\left(L_{2}-\left(\Delta_{i}-A_{i}\right)\right)$ is non-special; furthermore,

$$
\begin{aligned}
\operatorname{dim} W_{2, i} \geq 1+h^{0}\left(A_{i}, L_{2}-\left(\Delta_{i}-A_{i}\right)\right) & \\
& =p_{a}\left(A_{i}\right)+\left.\operatorname{deg}\left(L_{2}-K_{S}\right)\right|_{A_{i}}+\left(D-\Delta_{i}\right) A_{i}-D A_{i},
\end{aligned}
$$

since $\left|L_{2}\right|$ has no base points.

We exclude the possibility (i). Assume that $\Delta_{i}-\left(L_{2}-K_{S}\right)$ is nef on $\Delta_{i}$. Then we have $\Delta_{i} A \geq\left.\operatorname{deg}\left(L_{2}-K_{S}\right)\right|_{A} \geq K_{S} A \geq 0$ and, hence, $\left(D-\Delta_{i}\right) A \leq 0$ holds for any irreducible component $A$ of $\Delta_{i}$. Since $D$ is chain connected, this is possible only if $\Delta_{i}=D$. If $\Delta_{i}=D$, then we have $\left(D-\left(L_{2}-K_{S}\right)\right) D \geq 0$ and it would follow $D^{2} \geq 0$, which is absurd. Hence (i) is impossible, and we are in the case (ii). In particular, we must have $\left(D-\Delta_{i}\right) A_{i}>0$ except when $i=1$, $\Delta_{1}=D$.

We use (2.3) to examine (2.2). If $K_{A_{i}}+L_{2}-L_{1}+X_{N-i}$ is special on $A_{i}$, it suffices to check that $\left.\operatorname{deg}\left(L_{2}-K_{S}\right)\right|_{A_{i}}+\left(D-\Delta_{i}\right) A_{i}-D A_{i} \geq 2$, which is direct. If it is non-special on $A_{i}$, then a sufficient condition for (2.2) is that $2 p_{a}\left(A_{i}\right)+\left(D-\Delta_{i}\right) A_{i}-2 D A_{i}+D_{i-1} A_{i}+\left.\operatorname{deg}\left(L_{1}-2 K_{S}\right)\right|_{A_{i}} \geq 3$. This does not hold in the following cases:

(a) $i=1, \Delta_{1}=D, D A_{1}=-1, p_{a}\left(A_{1}\right)=\left.\operatorname{deg}\left(L_{1}-2 K_{S}\right)\right|_{A_{1}}=0$.

(b) $i \geq 2, p_{a}\left(A_{i}\right)=D A_{i}=\left.\operatorname{deg}\left(L_{1}-2 K_{S}\right)\right|_{A_{i}}=0,\left(D-\Delta_{i}\right) A_{i}=D_{i-1} A_{i}=1$.

In either case, $L_{1}, L_{2}$ and $2 K_{S}$ are of the same degree on $A_{i}$. 
Consider the case $(a)$. Recall that $D-\left(L_{2}-K_{S}\right)$ is nef on $D-A_{1}$. Since $L_{2}-2 K_{S}$ and $-D$ are both nef, we see that every component of $D-A_{1}$ is a (-2)-curve, $D\left(D-A_{1}\right)=0$ and $L_{2} \equiv 2 K_{S}$ on $D$. Consider the cohomology long exact sequence for

$$
0 \rightarrow \mathcal{O}_{D-A_{1}}\left(-A_{1}\right) \rightarrow \mathcal{O}_{D} \rightarrow \mathcal{O}_{A_{1}} \rightarrow 0 .
$$

We have $h^{0}\left(D, \mathcal{O}_{D}\right)=1$ and $H^{0}\left(D, \mathcal{O}_{D}\right) \rightarrow H^{0}\left(A_{1}, \mathcal{O}_{A_{1}}\right)$ is non-trivial. This shows that $H^{0}\left(D-A_{1}, \mathcal{O}\left(-A_{1}\right)\right)=0$. Recall that we have $\mathcal{O}_{D-A_{1}}\left(L_{2}\right) \simeq$ $\simeq \mathcal{O}_{D-A_{1}}$. It follows from the exact sequence

$$
0 \rightarrow H^{0}\left(D-A_{1}, L_{2}-A_{1}\right) \rightarrow H^{0}\left(D, L_{2}\right) \rightarrow H^{0}\left(A_{1}, L_{2}\right)
$$

and $H^{0}\left(D-A_{1}, L_{2}-A_{1}\right) \simeq H^{0}\left(D-A_{1}, \mathcal{O}_{D-A_{1}}\left(-A_{1}\right)\right)=0$ that $\operatorname{dim} W_{2,1}=$ $=h^{0}\left(D, L_{2}\right)=2 K_{S} D-D\left(K_{S}+D\right) / 2=(3 / 2) K_{S} A_{1}-D^{2} / 2$. Hence the inequality in (2.2) becomes $(1 / 2)\left(K_{S} A_{1}-D^{2}\right)-D A_{1} \geq 3$. This does not hold only when $K_{S} A_{1}=-D^{2}=-D A_{1}=1$, that is, $D$ is a $(-1)$ elliptic cycle on which $L_{2} \equiv 2 K_{S}$. We show that such a ( -1$)$ elliptic cycle $D$ is 2-connected. Let $D=A+B$ be any effective decomposition of $D$. We can assume that $A_{1}$ is contained in $A$. Then $B$ consists of ( -2$)$-curves and it follows that $B^{2}$ is a negative even integer. Recall that $\mathcal{O}_{D-A_{1}}(D)$ is numerically trivial. In particular, we have $0=D B=A B+B^{2}$. Hence we get $A B \geq 2$ and see that $D$ is 2-connected.

Consider the case (b). (2.2) is now $\operatorname{dim} W_{2, i} \geq-A_{i}^{2}=K_{S} A_{i}+2$. We have $\Delta_{i} A_{i}=-1$ and $\left(\Delta_{i}-A_{i}\right) \Delta_{i} \geq\left.\operatorname{deg}\left(L_{2}-K_{S}\right)\right|_{\Delta_{i}-A_{i}} \geq K_{S}\left(\Delta_{i}-A_{i}\right) \geq 0$. Since $0>\Delta_{i}^{2}=\left(\Delta_{i}-A_{i}\right) \Delta_{i}+A_{i} \Delta_{i} \geq K_{S}\left(\Delta_{i}-A_{i}\right)-1 \geq-1$, we get $\Delta_{i}^{2}=-1$. Then the equalities hold everywhere. Since $K_{S}\left(\Delta_{i}-A_{i}\right)=0, \Delta_{i}-A_{i}$ consists of ( -2$)$-curves. Furthermore, we see that $L_{2} \equiv 2 K_{S}$ on $\Delta_{i}$. We can show that $\Delta_{i}$ is 2-connected as in (a). Then $h^{0}\left(\Delta_{i}, \mathcal{O}_{\Delta_{i}}\right)=1$ and we get $\operatorname{dim} W_{2, i}=h^{0}\left(\Delta_{i}, L_{2}\right)=(3 / 2) K_{S} A_{i}+1 / 2$ similarly as in $(a)$. The desired inequality $\operatorname{dim} W_{2, i} \geq K_{S} A_{i}+2$ holds except when $K_{S} A_{i}=1$, that is, $\Delta_{i}$ is a Francia cycle of arithmetic genus one on which $L_{2} \equiv 2 K_{S}$.

Now we exchange the rôle of $L_{1}, L_{2}$, and consider the multiplication $\operatorname{map} \mu_{1, i}: W_{1, i} \otimes H^{0}\left(A_{i}, L_{2}-X_{N-i}\right) \rightarrow H^{0}\left(A_{i}, L_{1}+L_{2}-X_{N-i}\right)$ for each in$\operatorname{dex} i$ for which we failed to show the surjectivity of $\mu_{2, i}$. Since we can assume $\Delta_{1, i}=\Delta_{2, i}$ by the following lemma, the assertion follows.

Lemma 2.7. Let $S$ and $D$ be as in the previous theorem. If $E$ is a subcurve with $E^{2}=-1$, then the restriction map $H^{0}(D, L) \rightarrow H^{0}(E, L)$ is surjective for any line bundle $L$ on $D$ such that $L-2 K_{S}$ is nef.

Proof. We assume that the restriction map is not surjective and show that this leads us to a contradiction. Consider the cohomology long exact 
sequence for

$$
0 \rightarrow \mathcal{O}_{D-E}(L-E) \rightarrow \mathcal{O}_{D}(L) \rightarrow \mathcal{O}_{E}(L) \rightarrow 0 .
$$

Since $H^{1}(D, L)=0$, we must have $H^{1}(D-E, L-E) \neq 0$. By the duality, we get $H^{1}(D-E, L-E)^{\vee}=H^{0}\left(D-E, D-\left(L-K_{S}\right)\right)$. Let $s \in H^{0}(D-E, D-$ $\left.-\left(L-K_{S}\right)\right)$ be a non-zero element. If $Z$ is the biggest subcurve on which $s$ vanishes identically (possibly $Z=0$ ), then $D-\left(L-K_{S}\right)-Z$ is nef on $D-E-Z$. In particular, we have $(D-Z)(D-E-Z) \geq$ $\geq\left.\operatorname{deg}\left(L-K_{S}\right)\right|_{D-E-Z} \geq 0$. We have

$0 \leq(D-Z)(D-E-Z)=(D-Z)^{2}-E(D-Z)=(D-E-Z)^{2}+E(D-Z)-E^{2}$.

Since $(D-Z)^{2} \leq 0$ and $(D-E-Z)^{2}<0$, we get $E^{2} \leq E(D-Z) \leq 0$. Then we get $(D-E-Z)^{2}=-1, E(D-Z)=0$ and $(D-Z)(D-E-Z)=0$, because $E^{2}=-1$. This implies that $L-K_{S}$ is of degree zero on $D-E-Z$ and it follows that $K_{S}(D-E-Z)=0$, because $L-2 K_{S}$ is nef. This is impossible for the reason of parity.

\section{General degree bounds.}

From now on, $S$ denotes a non-singular projective minimal surface of general type. It is known that $2 K_{S}$ is free when $p_{g}(S)>0$ or $p_{g}=0, K_{S}^{2} \geq 5$ (see [7], [22], [2], [3]). In what follows, we always assume that $2 K_{S}$ is free. Put $r=P_{2}(S)-1=K_{S}^{2}+\chi\left(\mathcal{O}_{S}\right)-1$. Note that we have $r=1$ if and only if $p_{g}=q=0, K_{S}^{2}=1$, that is, $S$ is a numerical Godeaux surface. Since we have assumed that $2 K_{S}$ is free, we have $r \geq 2$ and therefore exclude Godeaux surfaces from our considerations. We also remark that the image of the bi-canonical map is necessarily a surface when $2 K_{S}$ is free.

Our main strategy in studying $R\left(S, K_{S}\right)$ is the use of the Koszul cohomology. The following two lemmas are easy applications of Theorems 1.1, 1.2 and 1.3, which combined with Lemma 1.4 can cover a major part of Theorem A.

Lemma 3.1. Suppose that $2 K_{S}$ is free. Then the multiplication map

$$
H^{0}\left(S, 2 K_{S}\right) \otimes H^{0}\left(S,(m-2) K_{S}\right) \rightarrow H^{0}\left(S, m K_{S}\right)
$$

is surjective in the following cases:

(1) $m>7$.

(2) $m=7$ and $K_{S}^{2}+p_{g}(S)-q(S) \geq 3$. 
(3) $m=6$ and $K_{S}^{2} \geq q(S)+2$.

(4) $m=5, q(S)=0$ and the bi-canonical image of $S$ is not a surface of minimal degree.

Proof. Since $S$ is a minimal surface of general type, we have $H^{1}\left(S, i K_{S}\right)=0$ when $i \neq 0,1$. Of course $H^{1}\left(S, i K_{S}\right)=0$ holds also for $i=0,1$ when $S$ is regular. Put $\varepsilon=0,1$ according to $m$ is even or odd. The multiplication map in question is surjective if and only if $K_{0,(m-\varepsilon) / 2}\left(S, \varepsilon K_{S}, 2 K_{S}, H^{0}\left(S, 2 K_{S}\right)\right)=0$. By the proof of Theorem 1.1 , the natural map

$$
\begin{aligned}
K_{0,(m-\varepsilon) / 2}\left(S, \varepsilon K_{S}, 2 K_{S}, H^{0}(S\right. & \left.\left., 2 K_{S}\right)\right) \\
& \rightarrow K_{r-2,3-(m-\varepsilon) / 2}\left(S,(1-\varepsilon) K_{S}, 2 K_{S}, H^{0}\left(S, 2 K_{S}\right)\right)^{\vee}
\end{aligned}
$$

is injective (resp. surjective) when $H^{1}\left(S,(m-4) K_{S}\right)=0$ (resp. $\left.H^{1}\left(S,(m-2) K_{S}\right)=0\right)$. Therefore, when $m \geq 6$ or $m=5, q(S)=0$, the problem is reduced to showing that the Koszul sequence

$$
\begin{aligned}
\bigwedge^{r-1} H^{0}\left(S, 2 K_{S}\right) \otimes H^{0}\left(S,(5-m) K_{S}\right) & \rightarrow \bigwedge^{r-2} H^{0}\left(S, 2 K_{S}\right) \otimes H^{0}\left(S,(7-m) K_{S}\right) \\
& \rightarrow \bigwedge^{r-3} H^{0}\left(S, 2 K_{S}\right) \otimes H^{0}\left(S,(9-m) K_{S}\right)
\end{aligned}
$$

is exact at the middle term. If $m \geq 8$, then $H^{0}\left(S,(7-m) K_{S}\right)=0$ and we have (1). If $m=7$, then we have $H^{0}\left(S,(5-m) K_{S}\right)=0, H^{0}\left(S,(7-m) K_{S}\right) \simeq \mathbb{C}$, and the Koszul map $\bigwedge^{r-2} H^{0}\left(S, 2 K_{S}\right) \rightarrow \bigwedge^{r-3} H^{0}\left(S, 2 K_{S}\right) \otimes H^{0}\left(S, 2 K_{S}\right)$ is injective when $r-3 \geq 0$, which gives (2). If $m=6$ and $p_{g}(S)=h^{0}\left(S, K_{S}\right) \leq$ $\leq r-2$, then the exactness follows from Theorem 1.2, which gives (3). Assume that $m=5$ and $q(S)=0$. Recall that the bi-canonical image is a surface, since $2 K_{S}$ is free. Then Theorem 1.3 shows that the above sequence is exact at the middle term, unless the bi-canonical image is a surface of minimal degree. Hence (4).

Quite similarly, we can show the following:

\section{LEMma 3.2. Assume that $2 K_{S}$ is free. Then the Koszul sequence}

$$
\bigwedge^{2} H^{0}\left(2 K_{S}\right) \otimes H^{0}\left((m-4) K_{S}\right) \rightarrow H^{0}\left(2 K_{S}\right) \otimes H^{0}\left((m-2) K_{S}\right) \rightarrow H^{0}\left(m K_{S}\right)
$$

is exact at the middle term in the following cases:

(1) $m>9$ 
(2) $m=9$ and $K_{S}^{2}+p_{g}(S)-q(S) \geq 4$,

(3) $m=8$ and $K_{S}^{2} \geq q(S)+3$.

Another strategy is the hyperplane section principle. Assume that $p_{g}(S)>0$ and let $D \in\left|K_{S}\right|$ be a general member. Put $L=K_{S \mid D}$. Consider the cohomology long exact sequence for

$$
0 \rightarrow \mathcal{O}_{S}\left(m K_{S}-D\right) \rightarrow \mathcal{O}_{S}\left(m K_{S}\right) \rightarrow \mathcal{O}_{D}(m L) \rightarrow 0
$$

for any non-negative integer $m$. Since $S$ is a minimal surface of general type, we have $H^{1}\left(S, m K_{S}-D\right)=H^{1}\left(S,(m-1) K_{S}\right)=0$ except when $q(S)>0$ and $m=1,2$. Hence the restriction map $H^{0}\left(S, m K_{S}\right) \rightarrow$ $\rightarrow H^{0}\left(D, m K_{S}\right)$ is surjective for $m \geq 0$ when $q(S)=0$ and for $m \neq 1,2$ when $q(S)>0$. Consider the homomorphism of graded C-algebras

$$
R\left(S, K_{S}\right)=\bigoplus_{m \geq 0} H^{0}\left(S, m K_{S}\right) \rightarrow R(D, L)=\bigoplus_{m \geq 0} H^{0}(D, m L) .
$$

induced by the restriction maps. The kernel is the homogeneous ideal of $R\left(S, K_{S}\right)$ generated by a single element in degree one, that is, a section defining $D$. Therefore, for example, in order to show that $R\left(S, K_{S}\right)$ is generated in degrees $\leq 3$ and related in degrees $\leq 6$, it is sufficient to show the same things for the image $R^{\prime}(D, L)$ of $R\left(S, K_{S}\right) \rightarrow R(D, L)$. Since $D$ is Gorenstein, Theorem 1.1 works well, when we study $R^{\prime}(D, L)$ via the Koszul cohomology groups.

Now, we are going to show Theorem A. Our basic assumption is that $2 K_{S}$ is free. Though a considerable part inevitably overlaps with [4] and [24], we do not exclude it for the convenience of readers, unless it involves too much. Among other things, we shall freely use basic inequalities in the surface geography: $K_{S}^{2}>0, \chi\left(\mathcal{O}_{S}\right)>0, K_{S}^{2} \geq 2 p_{g}(S)-4$, and $K_{S}^{2} \geq 2 p_{g}(S)$, when $q(S)>0$ (see [6]).

We study the exceptional cases in (2), (3), (4) of Lemma 3.1.

(a) Suppose that $K_{S}^{2}+p_{g}-q=2$. Then we have $K_{S}^{2}=2, p_{g}=q$; $K_{S}^{2}=1, p_{g}=q+1$. If $q(S)=0$, then $\left(p_{g}, K_{S}^{2}\right)=(0,2),(1,1)$. If $q(S)>0$, then $K_{S}^{2}=2$ and $p_{g}=q=1$, because $K_{S}^{2} \geq 2 p_{g}(S)$ holds for irregular surfaces of general type.

Lemma 3.3. Assume that $\left(p_{g}, q, K_{S}^{2}\right)=(0,0,2),(1,0,1),(1,1,2)$. Then the following hold:

(1) $\operatorname{corank}\left\{H^{0}\left(S, 2 K_{S}\right) \otimes H^{0}\left(S, 5 K_{S}\right) \rightarrow H^{0}\left(S, 7 K_{S}\right)\right\}=1$.

(2) $\operatorname{corank}\left\{H^{0}\left(S, 2 K_{S}\right) \otimes H^{0}\left(S, 4 K_{S}\right) \rightarrow H^{0}\left(S, 6 K_{S}\right)\right\}=p_{g}(S)$.

(3) $\operatorname{corank}\left\{H^{0}\left(S, 2 K_{S}\right) \otimes H^{0}\left(S, 3 K_{S}\right) \rightarrow H^{0}\left(S, 5 K_{S}\right)\right\}=3 q(S)$. 
Furthermore, the Koszul sequence

$\bigwedge^{2} H^{0}\left(S, 2 K_{S}\right) \otimes H^{0}\left(S,(m-4) K_{S}\right)$

$$
\rightarrow H^{0}\left(S, 2 K_{S}\right) \otimes H^{0}\left(S,(m-2) K_{S}\right) \rightarrow H^{0}\left(S, m K_{S}\right)
$$

is exact at the middle term for $m \geq 8$ when $q(S)=1$; for arbitrary $m$ when $q(S)=0$.

Proof. Let

$$
0 \rightarrow \mathcal{E} \rightarrow H^{0}\left(S, 2 K_{S}\right) \otimes \mathcal{O}_{S} \rightarrow \mathcal{O}_{S}\left(2 K_{S}\right) \rightarrow 0
$$

be the exact sequence obtained by the evaluation map. If $m \geq 4$, then we have $H^{1}\left(S,(m-2) K_{S}\right)=0$ and we see that the cokernel of $H^{0}\left(S, 2 K_{S}\right) \otimes H^{0}\left(S,(m-2) K_{S}\right) \rightarrow H^{0}\left(S, m K_{S}\right)$ is isomorphic to $H^{1}\left(S, \mathcal{E} \otimes \mathcal{O}_{S}\left((m-2) K_{S}\right)\right)$ which is dual to $H^{1}\left(S, \mathcal{E}^{\vee} \otimes \mathcal{O}_{S}\left((3-m) K_{S}\right)\right)$. Since $\bigwedge^{2} \mathcal{E} \simeq \mathcal{O}_{S}\left(-2 K_{S}\right)$, we have $\mathcal{E}^{\vee} \otimes \mathcal{O}_{S}\left((3-m) K_{S}\right) \simeq \mathcal{E}^{\vee} \otimes \bigwedge^{2} \mathcal{E} \otimes$ $\otimes \mathcal{O}_{S}\left((5-m) K_{S}\right) \simeq \mathcal{E} \otimes \mathcal{O}_{S}\left((5-m) K_{S}\right)$. So, we have only to calculate $h^{1}\left(S, \mathcal{E} \otimes \mathcal{O}_{S}\left((5-m) K_{S}\right)\right)$. It follows from the exact sequence

$$
\begin{array}{r}
H^{0}\left(S, 2 K_{S}\right) \otimes H^{0}\left(S,(5-m) K_{S}\right) \rightarrow H^{0}\left(S,(7-m) K_{S}\right) \rightarrow H^{1}\left(S, \mathcal{E} \otimes \mathcal{O}_{S}\left((5-m) K_{S}\right)\right) \\
\rightarrow H^{0}\left(S, 2 K_{S}\right) \otimes H^{1}\left(S,(5-m) K_{S}\right) \rightarrow H^{1}\left(S,(7-m) K_{S}\right)
\end{array}
$$

that we have

$$
H^{1}\left(S, \mathcal{E} \otimes \mathcal{O}_{S}(5-m) K_{S}\right) \simeq \begin{cases}H^{0}\left(S,(7-m) K_{S}\right) & \text { when } m=6,7 \\ H^{0}\left(S, 2 K_{S}\right) \otimes H^{1}\left(S, \mathcal{O}_{S}\right) & \text { when } m=5\end{cases}
$$

Hence we get (1)-(3). As to the last assertion, note the kernel of $H^{0}\left(S, 2 K_{S}\right) \otimes H^{0}\left(S,(m-2) K_{S}\right) \rightarrow H^{0}\left(S, m K_{S}\right)$ is $H^{0}\left(S, \mathcal{E} \otimes \mathcal{O}_{S}\left((m-2) K_{S}\right)\right)$. Since $\bigwedge^{2} \mathcal{E} \simeq \mathcal{O}_{S}\left(-2 K_{S}\right)$ and we have the exact sequence

$$
\begin{aligned}
0 \rightarrow \bigwedge^{2} \mathcal{E} \otimes \mathcal{O}_{S}\left((m-4) K_{S}\right) \rightarrow \bigwedge^{2} H^{0}\left(S, 2 K_{S}\right) \otimes & \mathcal{O}_{S}\left((m-4) K_{S}\right) \\
& \rightarrow \mathcal{E} \otimes \mathcal{O}_{S}\left((m-2) K_{S}\right) \rightarrow 0
\end{aligned}
$$

we have only to check whether $H^{1}\left(S,(m-6) K_{S}\right)$ vanishes or not.

When $K_{S}^{2}=p_{g}=1$ and $q=0$, it is known [1] that the canonical model of $S$ is isomorphic to a complete intersection of type $(6,6)$ in the weighted projective space $\mathrm{P}(1,2,2,3,3)$. Therefore, $R\left(S, K_{S}\right)$ is generated in degrees $\leq 3$ and related in degrees $\leq 6$. We study the other two cases. 
Lemma 3.4. Assume that $K_{S}^{2}=2$ and $p_{g}=q=0$. If $2 K_{S}$ is free, then $R\left(S, K_{S}\right)$ is generated in degrees $\leq 4$ and related in degrees $\leq 8$.

Proof. We shall show that $H^{0}\left(S, 3 K_{S}\right) \otimes H^{0}\left(S, 4 K_{S}\right) \rightarrow H^{0}\left(S, 7 K_{S}\right)$ is surjective. Note that $3 K_{S}$ is free (see, e.g., [22]). By Theorem 1.1, it suffices to show that

$$
\bigwedge^{5} H^{0}\left(S, 3 K_{S}\right) \rightarrow \bigwedge^{4} H^{0}\left(S, 3 K_{S}\right) \otimes H^{0}\left(S, 3 K_{S}\right) \rightarrow \bigwedge^{3} H^{0}\left(S, 3 K_{S}\right) \otimes H^{0}\left(S, 6 K_{S}\right)
$$

is exact at the middle term. By Theorem 1.3, we have the claim unless the tri-canonical image of $S$ is a surface of degree 5 in $\mathbb{P}^{6}$. Since we have $\left(3 K_{S}\right)^{2}=18$ which is not a multiple of 5 , we are done. This and Lemma 3.3 show that $R\left(S, K_{S}\right)$ is generated in degrees $\leq 4$. Then putting $d=4, d_{0}=7$, $d_{1}=0$ and $k=2$, Lemma 1.4 shows that it is related in degrees $\leq 10=4+7-1$. Since $p_{g}=0$, we have no generators in degree 1 . If there is a relation of degree 9 or 10, then any monomial appearing the relation can be divided by a monomial of degree 5 or 6 . Then, using Lemma 3.3, the proof of Lemma 1.4 shows that such a relation can be reduced to relations in degrees $\leq 8$.

Lemma 3.5. Assume that $K_{S}^{2}=2, p_{g}=q=1$. Then $R\left(S, K_{S}\right)$ is generated in degrees $\leq 5$ and related in degrees $\leq 10$.

Proof. Surfaces with such numerical invariants are extensively studied in [12]. We freely use the results there. The Albanese map $f: S \rightarrow B=\operatorname{Alb}(S)$ is a fibration of genus 2. Then $f_{*} \omega_{S}$ is an indecomposable, nef locally free sheaf of rank two and of degree one. Since it has no locally free quotient of degree zero, we have an exact sequence

$$
0 \rightarrow \mathcal{O}_{B} \rightarrow f_{*} \omega_{S} \rightarrow \mathcal{O}_{B}(P) \rightarrow 0,
$$

for a point $P \in B$. By [12], $S$ is obtained as the minimal resolution of a finite double covering surface of $\mathbb{P}=\mathbb{P}_{B}\left(f_{*} \omega_{S}\right)$ which has at most rational double points and with branch locus in $|6 \Delta-2 \Gamma|$, where $\Delta$ is a tautological divisor and $\Gamma$ is the fibre of $\mathbb{P} \rightarrow B$ over $P$. Hence $K_{S}$ is induced by $\Delta$. Furthermore, we have the decomposition $H^{0}\left(S, m K_{S}\right) \simeq H^{0}(\mathbb{P}, m \Delta) \oplus H^{0}(\mathbb{P},(m-3) \Delta+\Gamma)$ into the $( \pm 1)$-eigen spaces under the action of the covering transformation group.

We first claim that the invariant part $\bigoplus_{m>0} H^{0}(\mathrm{P}, m \Delta)$ is generated in degrees $\leq 4$. We show that $H^{0}(\mathbb{P}, 2 \Delta) \otimes H^{0}(\overline{\mathbb{P}},(m-2) \Delta) \rightarrow H^{0}(\mathbb{P}, m \Delta)$ is surjective for $m \geq 5$. By the duality theorem, it suffices to show that 
$H^{1}\left(\mathbb{P},(6-m) \Delta+K_{\mathrm{P}}\right)=0$. Since the canonical bundle of $\mathbb{P}$ is given by $-2 \Delta+\Gamma$, we are done. As to the anti-invariant part, we consider

$$
\bigoplus_{i=1}^{m-3} H^{0}(\mathbb{P}, i \Delta) \otimes H^{0}(\mathbb{P},(m-3-i) \Delta+\Gamma) \rightarrow H^{0}(\mathbb{P},(m-3) \Delta+\Gamma) .
$$

Note that the image of $H^{0}(\Delta) \otimes H^{0}((m-4) \Delta+\Gamma)$ consists of those sections vanishing on $\Delta$, whilst that of $H^{0}((m-3) \Delta) \otimes H^{0}(\Gamma)$ consists of sections vanishing on $\Gamma$. In particular, when $m=5$, this implies that we need a new generator in degree 5 which does not vanish at $\Delta \cap \Gamma$. If $m \geq 6$, then the image of $H^{0}(2 \Delta) \otimes H^{0}((m-5) \Delta+\Gamma)$ contains a section not vanishing at $\Delta \cap \Gamma$. This and Lemma $3.3 \mathrm{imply}$ that $R\left(S, K_{S}\right)$ is generated in degrees $\leq 5$.

It remains to show that $R\left(S, K_{S}\right)$ is related in degrees $\leq 10$. By Lemma 1.4 with $d=5, d_{0}=d_{1}=7$ and $k=2$, we see that it is related in degrees $\leq 11=5+7-1$. Assume that we have a relation in degree 11 . Let $G$ be a monomial of degree 11 appearing in the relation. Then it is easy to see that $G$ can be divided by a monomial $G_{1}$ of degree 8 or 9 . Hence, as in the proof of Lemma 1.4, we can show that any relations in degree 11 are induced by relations in lower degrees.

(b) Suppose that $K_{S}^{2} \leq q(S)+1$ but $K_{S}^{2}+p_{g}-q \geq 3$. By Noether's inequality and the fact that $K_{S}^{2} \geq 2 p_{g}$ holds for irregular surfaces, we get $p_{g}=2, q=0, K_{S}^{2}=1$. It is known [11] that the canonical model of a surface with $p_{g}=2, q=0, K_{S}^{2}=1$ is isomorphic to a hypersurface of degree $10 \mathrm{in}$ $\mathbb{P}(1,1,2,5)$. In particular, $R\left(S, K_{S}\right)$ is generated in degrees $\leq 5$ and related in degrees $\leq 10$.

(c) Suppose that $q(S)=0, K_{S}^{2}+p_{g} \geq 3, K_{S}^{2} \geq 2$ and that the bi-canonical image of $S$ is a surface of minimal degree $r-1$ in $\mathbb{P}^{r}$. By the classification [18], a surface of minimal degree is either $(a) \mathbb{P}^{2}(r=2)$, or $(b)$ a quadric surface in $\mathbb{P}^{3}(r=3)$, or $(c)$ the Veronese surface in $\mathbb{P}^{5}(r=5)$, or $(d)$ a rational normal surface scroll. In particular, it is ruled by straight lines except when it is the Veronese surface. If $k$ denotes the degree of the bicanonical map (onto the image), then

$$
4 K_{S}^{2}=k\left(K_{S}^{2}+p_{g}-1\right) \text {. }
$$

We divide the case into sub-cases according to $k$.

(c1) If $k=2$, then $K_{S}^{2}=p_{g}-1$. It follows $\left(K_{S}^{2}, p_{g}\right)=(2,3)$. Then it is shown in [10] that the canonical model of $S$ is isomorphic to a hypersurface of degree 8 in $\mathbb{P}(1,1,1,4)$. In particular, $R\left(S, K_{S}\right)$ is generated in degrees $\leq 4$ and related in degrees $\leq 8$. 
(c2) If $k=3$, then we have $K_{S}^{2}=3 p_{g}-3$. Assume that the bi-canonical image is ruled by straight lines and let $D$ be the pull-back to $S$ of a line. Then, since the bi-canonical map is of degree 3 , we must have $2 K_{S} D=3$, which is absurd. Assume that the bi-canonical image is the Veronese surface in $\mathbb{P}^{5}$. Then we have $p_{g}(S)=2, q(S)=0$ and $K_{S}^{2}=3$. Furthermore, we have a divisor $H$ with $2 K_{S}=2 H$ coming from a line on $\mathbb{P}^{2}$. Note that we have $H \neq K_{S}$, because $h^{0}\left(S, K_{S}\right)=2$ but $h^{0}(S, H) \geq h^{0}\left(\mathbb{P}^{2}, \mathcal{O}_{\mathrm{P}}(1)\right)=3$. In particular, $S$ is not simply connected and the unramified double covering $\tilde{S}$ of $S$ associated to the 2 -torsion divisor $K_{S}-H$ is a surface with $p_{g}=5$ and $K^{2}=6$. Now, $\tilde{S}$ is on the Noether line and, therefore, has a unique pencil of curves of genus 2 (see, [10]). By the uniqueness, the pencil is preserved by the action of the covering transformation group of $\tilde{S} \rightarrow S$. This implies that $S$ also has a pencil of curves of genus 2. Then the bi-canonical map of $S$ cannot be of odd degree, since it factors through the relative bi-canonical map which is of degree 2 . Hence such a surface does not exist.

(c3) If $k=4$, then we have $p_{g}=1$. We assume that $K_{S}^{2} \geq 5$ and show that this leads us to a contradiction. We have $r \geq 6$ and it follows that the bicanonical image is ruled by lines. Then we have a pencil $|D|$ of curves with $2 K_{S} D=4$, that is, $K_{S} D=2$. Hodge's index theorem shows that $4=\left(K_{S} D\right)^{2} \geq K_{S}^{2} D^{2}$. Since $K_{S}^{2} \geq 5$, we get $D^{2}=0$. Then $|D|$ is a pencil of curves of genus 2. This is impossible by Xiao's theorem [23, Théorème 5.6] which in particular implies that $S$ does not have a pencil of curves of genus 2 when $K_{S}^{2} \geq 5$ and the degree of the bi-canonical map is 4 . Hence $K_{S}^{2} \leq 4$.

Lemma 3.6. Let $S$ be a minimal regular surface of general type with $p_{g}(S)=1,2 \leq K_{S}^{2} \leq 4$. Then $R\left(S, K_{S}\right)$ is generated in degrees $\leq 4$ and related in degrees $\leq 8$.

Proof. Since $p_{g}=1$, we have a unique canonical curve $D \in\left|K_{S}\right|$. We show that the multiplication map $H^{0}\left(S, 2 K_{S}\right) \otimes H^{0}\left(S, 3 K_{S}\right) \oplus H^{0}\left(S, K_{S}\right) \otimes$ $\otimes H^{0}\left(S, 4 K_{S}\right) \rightarrow H^{0}\left(S, 5 K_{S}\right)$ is surjective. For this purpose, it is sufficient to show that $H^{0}(D, 2 L) \otimes H^{0}(D, 3 L) \rightarrow H^{0}(D, 5 L)$ is surjective, where $L=\left.K_{S}\right|_{D}$ (see the argument around (3.4)). By Theorem 1.1, it is surjective when

$$
\bigwedge_{K_{S}^{2}-1} H^{0}(D, 2 L) \otimes H^{0}(D, L) \rightarrow \bigwedge_{K_{S}^{2}-2} H^{0}(D, 2 L) \otimes H^{0}(D, 3 L)
$$

is injective. Since $p_{g}=1$ and $q=0$, we have $h^{0}(D, L)=0$. Therefore, $R\left(S, K_{S}\right)$ is generated in degrees $\leq 4$.

In order to see that $R(D, L)$ (hence also $R\left(S, K_{S}\right)$ ) is related in degrees 
$\leq 8$, by Lemma 1.4, it is sufficient to show that the Koszul sequence

$\bigwedge^{2} H^{0}(D, 2 L) \otimes H^{0}(D,(m-4) L) \rightarrow H^{0}(D, 2 L) \otimes H^{0}(D,(m-2) L) \rightarrow H^{0}(D, m L)$

is exact at the middle term for $m \geq 9$. By Theorem 1.1, we only have to show that

$$
\bigwedge_{K_{S}^{2}-2} H^{0}(D, 2 L) \otimes H^{0}(D,(8-m) L) \rightarrow \bigwedge^{K_{S}^{2}-3} H^{0}(D, 2 L) \otimes H^{0}(D,(6-m) L)
$$

is injective for $m \geq 9$. Since $H^{0}(D,(8-m) L)=0$ unless $m=8$, we are done.

(c4) If $k \geq 5$, then $p_{g}=0$ and $\left(k, K_{S}^{2}\right)=(5,5)$ or $(6,3)$. As Mendes Lopes showed in [17], both cases are impossible.

We must take into account one more case in which Lemma 3.2, (2) does not hold: $p_{g}=q=0$ and $K_{S}^{2}=3$. In this case, we already know that $R\left(S, K_{S}\right)$ is generated in degrees $\leq 4$. By Lemmas 3.2 and 1.4, it is related in degrees $\leq 9$.

Now, Theorem A in Introduction follows from Lemmas 3.1, 3.2, 1.4 and what we showed above.

REMARK 3.7. In Theorem A, the assertion for generators were already shown by Ciliberto [4]. See also [17] for surfaces with $p_{g}=q=0$. As to surfaces with $p_{g}=q=0$ and $K_{S}^{2}=3$, one can show that $R\left(S, K_{S}\right)$ is related in degrees $\leq 8$ if a general $C \in\left|2 K_{S}\right|$ is nontrigonal. In fact, it is known that the tri-canonical map of $S$ is a birational morphism. Since $K_{C}=\left.3 K_{S}\right|_{C}$, it follows that $C$ is non-hyperelliptic. So, if $C$ is non-trigonal, then the canonical ring of $C$ is related in degree 2 and, hence,

$$
\stackrel{2}{\bigwedge}^{0} H^{(}\left(C, K_{C}\right) \otimes H^{0}\left(C, K_{C}\right) \rightarrow H^{0}\left(C, K_{C}\right) \otimes H^{0}\left(C, 2 K_{C}\right) \rightarrow H^{0}\left(C, 3 K_{C}\right)
$$

is exact at the middle term. Noting that $K_{C}=\left.3 K_{S}\right|_{C}$, one sees that $R\left(C,\left.K_{S}\right|_{C}\right)$ is related in degrees $\leq 8$. Then $R\left(S, K_{S}\right)$ is also related in degrees $\leq 8$, since the kernel of the surjection $R\left(S, K_{S}\right) \rightarrow R\left(C,\left.K_{S}\right|_{C}\right)$ is a principle ideal generated by an element in degree 2 . We do not know, however, whether a general bi-canonical curve is trigonal or not. 


\section{The case of rational fixed part.}

In this section and the next one, we shall show Theorem B. We consider in this section (1) of Theorem B.

We let $S$ be a minimal surface of general type with $p_{g}(S) \geq 2$ and $q(S)=0$. Let $\left|K_{S}\right|=|M|+Z$ be the decomposition of $\left|K_{S}\right|$ into the variable part $|M|$ and the fixed part $Z$. We denote by $C \in|M|$ a general member. Then $C$ is a reduced Gorenstein curve of arithmetic genus $M^{2}+1+M Z / 2$. We put $D=C+Z$. Then $D \in\left|K_{S}\right|$. Since $K_{S}$ is nef and big, and since $K_{S} A+A^{2}$ is an even integer for any curve $A$ on $S$, we see that $D$ is a numerically 2-connected curve.

Lemma 4.1. Let $\Gamma \preceq Z$ be a subcurve with $(Z-\Gamma) \Gamma=0$. Then $M \Gamma$ is a non-negative even integer. Furthermore, $M \Gamma=0$ if and only if $\Gamma=0$.

Proof. Since $K_{S} \Gamma+\Gamma^{2}$ is even and $K_{S} \Gamma+\Gamma^{2}=M \Gamma+2 \Gamma^{2}+$ $+(Z-\Gamma) \Gamma=M \Gamma+2 \Gamma^{2}$, we see that $M \Gamma$ is a non-negative even integer. Assume that $M \Gamma=0$. Then we have $0 \leq K_{S} \Gamma=M \Gamma+\Gamma^{2}+$ $+(Z-\Gamma) \Gamma=\Gamma^{2}$. If $M^{2}>0$, then it follows from Hodge's index theorem that $\Gamma^{2} \leq 0$. Hence $\Gamma^{2}=0$ and we get $\Gamma=0$. If $M^{2}=0$, then the canonical map of $S$ is composed of a pencil and it follows from $M \Gamma=0$ that every component of $\Gamma$ is vertical. Since the intersection form is negative semi-definite on fibres by Zariski's lemma, we have $\Gamma^{2} \leq 0$ implying $\Gamma^{2}=0$. Since $K_{S} \Gamma=0$ and $K_{S}^{2}>0$, we get $\Gamma=0$ by Hodge's index theorem.

We put $L=K_{S \mid D}$. Then $L$ is nef and we have $2 L=K_{D}$ by the adjunction formula. The restriction map $H^{0}\left(S, m K_{S}\right) \rightarrow H^{0}(D, m L)$ is surjective for any non-negative integer $m$, since $q(S)=0$.

LEMma 4.2. If $K_{S}^{2} \geq 2$, then $|2 L|$ and $|3 L|$ are free from base points.

Proof. Since $p_{g}(S)>0$, the bi-canonical system $\left|2 K_{S}\right|$ is free from base points by Francia's theorem. Hence $\left|K_{D}\right|=|2 L|$ is also free from base points. We have $p_{a}(D)=K_{S}^{2}+1$ and $\operatorname{deg} L=K_{S}^{2}$. It follows that $3 L$ may have base points only when $K_{S}^{2}=1$ (see, e.g., [13]).

Note that the restriction map $H^{0}(D, L) \rightarrow H^{0}(Z, L)$ is the zero map, because $Z$ is the fixed part of $\left|K_{S}\right|$. Consider the cohomology long exact 
sequence for

$$
0 \rightarrow \mathcal{O}_{S} \rightarrow \mathcal{O}_{S}(Z) \rightarrow \mathcal{O}_{Z}(Z) \rightarrow 0 .
$$

Since we have $h^{0}\left(S, \mathcal{O}_{S}(Z)\right)=1$ and $h^{1}\left(S, \mathcal{O}_{S}\right)=0$, we obtain $h^{0}\left(Z, \mathcal{O}_{Z}(Z)\right)=0$.

A numerically 1-connected curve with self-intersection number -2 is called a ( -2$)$-cycle, if it consists of $(-2)$-curves.

Lemma 4.3. Let $\left|K_{S}\right|=|M|+Z$ be the decomposition as above. If $\Gamma \preceq Z$ is a subcurve satisfying $M \Gamma=2,(Z-\Gamma) \Gamma=0$ and $\Gamma^{2} \leq 0$, then either $\Gamma$ is $a(-2)$-cycle or it contains a Francia cycle.

Proof. Since $D$ is numerically 2-connected and $(D-\Gamma) \Gamma=$ $=(C+(Z-\Gamma)) \Gamma=2$, we see that $\Gamma$ is numerically 1-connected. We have $0 \leq K_{S} \Gamma=M \Gamma+\Gamma^{2}=2+\Gamma^{2}$. Assume that $\Gamma^{2}=-2$. Then $p_{a}(\Gamma)=0$ and $\Gamma$ consists of $(-2)$-curves because $K_{S} \Gamma=0$. Hence $\Gamma$ is a $(-2)$-cycle. Assume that $\Gamma^{2}=-1$. Then $p_{a}(\Gamma)=1$. If $\Gamma$ is numerically 2-connected, then $\Gamma$ is a Francia cycle. If $\Gamma$ is not 2-connected, we get an effective decomposition $\Gamma=A+B$ with $A B=1$. Then $A, B$ are both 1 -connected and we have $1=p_{a}(\Gamma)=p_{a}(A)+p_{a}(B)-1+A B=p_{a}(A)+p_{a}(B)$. Hence either $p_{a}(A)=1$ or $p_{a}(B)=1$. Assume that $p_{a}(A)=1$. If $A$ is not 2-connected, we argue as above with $A$ instead of $\Gamma$ to find a 1 -connected subcurve $A^{\prime}$ with $p_{a}\left(A^{\prime}\right)=1$. Therefore, without loosing generality, we may assume that $A$ is 2-connected. We have $K_{S} A+A^{2}=0$ and $1=K_{S} \Gamma \geq K_{S} A$. It follows $K_{S} A=1$ and $A$ is a Francia cycle. Assume finally that $\Gamma^{2}=0$. Then $p_{a}(\Gamma)=2$ and $\Gamma$ is a Francia cycle of arithmetic genus two if it is numerically 2-connected. If $\Gamma$ is not 2-connected, letting $\Gamma=A+B$ be an effective decomposition such that $A B=1$ and $p_{a}(A) \geq p_{a}(B)$, we get $p_{a}(A)+p_{a}(B)=2$ and $A, B$ are 1-connected. Then $\left(p_{a}(A), p_{a}(B)\right)=$ $=(1,1),(2,0)$. We also have $2=K_{S} \Gamma=K_{S} A+K_{S} B$ and $A^{2}+B^{2}=-2$. When $p_{a}(A)=1$, we may assume that $A$ is 2 -connected as in the previous case to conclude that $A$ is a Francia cycle of arithmetic genus one. Assume that $p_{a}(A)=2$. If $K_{S} A=1$, then $A^{2}=1$ and $1=\left(K_{S} A\right)^{2} \geq K_{S}^{2} A^{2}=K_{S}^{2}$ by Hodge's index theorem. So, $K_{S}^{2}=1$ and $A$ is numerically equivalent to $K_{S}$. In particular, $A$ is nef. It would follow $1=K_{S} A=M A+(Z-\Gamma) A+A \Gamma \geq$ $\geq A^{2}+A B=2$, which is absurd. Hence, $K_{S} A=2$ and $A^{2}=0$ when $p_{a}(A)=2$. If $A$ is 2-connected, then it is a Francia cycle. If $A$ is not 2-connected, then we can repeat the above argument for $A$ instead of $\Gamma$.

The following is a direct consequence of [16, Theorem 4.2]. 
LEMMA 4.4. With the above notation, assume that $C$ is an irreducible curve and that $Z$ does not contain a Francia cycle. If the bi-canonical map of $S$ does not map $C$ birationally onto the image, then $C$ is a hyperelliptic curve, that is, C has a $g_{2}^{1}$. If furthermore either $M^{2} \geq 5$ or $h^{0}\left(Z, K_{Z}\right) \leq 2$ or $\mathcal{O}_{Z}(-Z)$ is nef, then $Z$ consists of at most $(-2)$-cycles.

Proof. We have $p_{a}(C) \geq 2$ because $S$ is of general type and $C$ moves in $|M|$. Recall that $D$ is 2-connected.

Suppose that there are points $x, y \in C$ which are non-singular points of $D$ (possibly $x=y$ ) and such that $x, y$ are not separated by $\left|K_{D}\right|$. Then, by [16, Theorem 4.2], we have the following possibilities:

(a) $D$ is an irreducible hyperelliptic curve and $\left|\mathcal{O}_{D}(x+y)\right|$ is the unique $g_{2}^{1}$ on $D$.

(b) $D$ is reducible, $C$ is a hyperelliptic curve, and $D$ decomposes as a sum $C+F_{1}+\cdots+F_{m}$ satisfying:

(i) $F_{1}, \ldots, F_{m}$ are curves such that $C F_{i}=2$ for every $i \in\{1, \ldots, m\}$.

(ii) $\mathcal{O}_{C}\left(F_{i}\right) \simeq \mathcal{O}_{C}(x+y)$ for every $i \in\{1, \ldots, m\}$.

(iii) $\left|\mathcal{O}_{C}(x+y)\right|$ is a $g_{2}^{1}$ on $C$.

(iv) $F_{i} F_{j}=0$ for $i \neq j$.

(v) $\mathcal{O}_{F_{i}}\left(F_{k}\right) \simeq \mathcal{O}_{F_{i}}$ for all $k<i$.

If we are in case $(a)$, then we have nothing to prove. Suppose that we are in case $(b)$. We have $F_{i} \preceq Z$ and $\left(Z-F_{i}\right) F_{i}=0$ for each $i$. Since $Z$ does not contains a Francia cycle, it suffices to show that $F_{i}^{2} \leq 0$ by the previous lemma. If $M^{2} \geq 5$, then we get $F_{i}^{2} \leq 0$, because we have $4=\left(M F_{i}\right)^{2} \geq$ $\geq M^{2} F_{i}^{2}$ by Hodge's index theorem. If $h^{0}\left(Z, K_{Z}\right) \leq 2$, then we have $p_{a}\left(F_{i}\right) \leq 2$ and it follows that $F_{i}^{2} \leq 0$. If $\mathcal{O}_{Z}(-Z)$ is nef, then we automatically have $F_{i}^{2} \leq 0$.

Note that the condition that $C$ is irreducible is equivalent to that the canonical map of $S$ is generically finite onto the image, when $p_{g}(S) \geq 3$. From now on, we assume that $C$ is an irreducible curve.

Lemma 4.5. Assume that $C$ is an irreducible curve. For a positive integer $p$, the Koszul map

$$
\bigwedge^{p} H^{0}(D, 2 L) \otimes H^{0}(D, L) \rightarrow \bigwedge^{p-1} H^{0}(D, 2 L) \otimes H^{0}(D, 3 L)
$$

is injective if $h^{0}(D, L) \leq p-h^{0}\left(Z, K_{Z}\right)$. 
Proof. Recall that we have $h^{0}\left(Z, \mathcal{O}_{Z}(Z)\right)=0$. Then, it follows from the exact sequence

$$
0 \rightarrow H^{0}\left(Z, \mathcal{O}_{Z}(Z)\right) \rightarrow H^{0}(D, L) \rightarrow H^{0}(C, L)
$$

that the restriction map $H^{0}(D, L) \rightarrow H^{0}(C, L)$ is injective.

Put $r=h^{0}(D, 2 L)-1=K_{S}^{2}$ and let $W$ be the image of the restriction map $H^{0}(D, L) \rightarrow H^{0}(C, L)$. From the exact sequence

$0 \rightarrow H^{0}\left(Z, K_{Z}\right) \rightarrow H^{0}(D, 2 L) \rightarrow H^{0}(C, 2 L) \rightarrow H^{1}\left(Z, K_{Z}\right) \rightarrow H^{1}(D, 2 L) \rightarrow 0$,

we get $\operatorname{dim} W=r+1-h^{0}\left(Z, K_{Z}\right)$. We choose a basis $\left\{s_{0}, s_{1}, \ldots, s_{r}\right\}$ of $H^{0}(D, 2 L)$ such that $s_{i}$ vanishes identically on $C$ for $r+1-h^{0}\left(Z, K_{Z}\right) \leq$ $\leq i \leq r$ and the rest induce a basis for $W$. We take sufficiently general points $P_{0}, \ldots, P_{r-h^{0}\left(Z, K_{Z}\right)}$ on $C$ so that $s_{i}\left(P_{j}\right)=\delta_{i, j}$. We consider when the Koszul map

$$
\bigwedge^{p} H^{0}(D, 2 L) \otimes H^{0}(D, L) \rightarrow \bigwedge^{p-1} H^{0}(D, 2 L) \otimes H^{0}(D, 3 L)
$$

is injective. The map can be identified with

$$
\bigwedge^{r+1-p} H^{0}(D, 2 L)^{\vee} \otimes H^{0}(D, L) \rightarrow \bigwedge^{r+2-p} H^{0}(D, 2 L)^{\vee} \otimes H^{0}(D, 3 L) .
$$

Put $t=r+1-p$ and let $\left\{e_{0}, e_{1}, \ldots, e_{r}\right\}$ be the dual basis for $H^{0}(D, 2 L)^{\vee}$. Then the last map is given by

$$
\sum \alpha_{i_{1}, \ldots, i_{t}} e_{i_{1}} \wedge \cdots \wedge e_{i_{t}} \mapsto \sum(-1)^{j} \alpha_{i_{1}, \ldots, \hat{i}_{j}, \ldots, i_{t+1}} s_{i_{j}} e_{i_{1}} \wedge \cdots \wedge e_{i_{t+1}} .
$$

Therefore, an element in the kernel can be identified with a collection $\left\{\alpha_{i_{1}, \ldots, i_{t}}\right\} \subset H^{0}(D, L)$ such that

$$
\alpha_{i_{2}, \ldots, i_{t+1}} s_{i_{1}}+\cdots+\alpha_{i_{1}, \ldots, i_{t}} s_{i_{t+1}}=0 .
$$

Evaluating at $P_{j}$, we see that $\alpha_{i_{1}, \cdots, i_{t}}\left(P_{j}\right)=0$ for any $j \in\{0, \cdots, r-$ $\left.-h^{0}\left(Z, K_{Z}\right)\right\} \backslash\left\{i_{1}, \ldots, i_{t}\right\}$. Hence, if $\operatorname{dim} \operatorname{Im}\left\{H^{0}(D, L) \rightarrow H^{0}(C, L)\right\} \leq$ $\leq r+1-h^{0}\left(Z, K_{Z}\right)-t=p-h^{0}\left(Z, K_{Z}\right)$, then $\alpha_{i_{1}, \ldots, i_{t}}$ vanishes identically on $C$. Since the restriction map $H^{0}(D, L) \rightarrow H^{0}(C, L)$ is injective, we conclude that $\alpha_{i_{1}, \ldots, i_{t}}=0$ in $H^{0}(D, L)$. Therefore, the Koszul map in question is injective when $h^{0}(D, L) \leq p-h^{0}\left(Z, K_{Z}\right)$.

Lemma 4.6. Assume that $p_{g}(S) \geq 2$ and $C$ is an irreducible curve. If $K_{S}^{2} \geq p_{g}(S)+1+h^{0}\left(Z, K_{Z}\right)$, then

$$
H^{0}(D, 2 L) \otimes H^{0}(D,(m-2) L) \rightarrow H^{0}(D, m L)
$$


is surjective for $m \geq 5$, and

$\bigwedge^{2} H^{0}(D, 2 L) \otimes H^{0}(D,(m-4) L) \rightarrow H^{0}(D, 2 L) \otimes H^{0}(D,(m-2) L) \rightarrow H^{0}(D, m L)$

is exact at the middle term for $m \geq 7$.

Proof. By Theorem 1.1, we only have to check that

$$
\bigwedge^{r-1} H^{0}(D, 2 L) \otimes H^{0}(D,(6-m) L) \rightarrow \bigwedge^{r-2} H^{0}(D, 2 L) \otimes H^{0}(D,(8-m) L)
$$

and

$$
\bigwedge^{r-2} H^{0}(D, 2 L) \otimes H^{0}(D,(8-m) L) \rightarrow \bigwedge^{r-3} H^{0}(D, 2 L) \otimes H^{0}(D,(10-m) L)
$$

are injective for $m \geq 5$ and $m \geq 7$, respectively, where $r=K_{S}^{2}$. Since $r \geq 3$, this is clear except in the cases $m=5$ and $m=7$, respectively. The rest follows from the previous lemma.

Lemma 4.7. If $H^{0}\left(Z, K_{Z}\right)=0$ and the bi-canonical map of $S$ maps $C$ birationally onto the image, then $H^{0}(D, 2 L) \otimes H^{0}(D, 2 L) \rightarrow H^{0}(D, 4 L)$ is surjective.

Proof. By Theorem 1.1, we only have to check that

$$
\bigwedge^{r} H^{0}(D, 2 L) \rightarrow \bigwedge^{r-1} H^{0}(D, 2 L) \otimes H^{0}(D, 2 L) \rightarrow \bigwedge^{r-2} H^{0}(D, 2 L) \otimes H^{0}(D, 4 L)
$$

is exact at the middle term, where $r=K_{S}^{2}=h^{0}(D, 2 L)-1$. Recall that $K_{D}=2 L$. Consider the exact sequence

$$
0 \rightarrow H^{0}\left(Z, K_{Z}\right) \rightarrow H^{0}\left(D, K_{D}\right) \rightarrow H^{0}\left(C, K_{D}\right) \rightarrow H^{1}\left(Z, K_{Z}\right) .
$$

If $H^{0}\left(Z, K_{Z}\right)=0$, then the restriction map $H^{0}\left(D, K_{D}\right) \rightarrow H^{0}\left(C, K_{D}\right)$ is injective. This implies that the image $C^{\prime}$ of $C$ under the canonical map of $D$ (or, the bi-canonical map of $S$ ) is an irreducible non-degenerate curve in $\mathbb{P} H^{0}(D, 2 L)$. Therefore, if the above Koszul complex is not exact at the middle term, we can apply [8, Theorem 1.13] (or the $K_{p, 1}$ theorem) to conclude that $C^{\prime}$ is a rational normal curve. This is absurd, because $C$ is not a rational curve.

Assume that $Z$ supports at most rational double points (possibly $Z=0$ ). Then, by contracting $Z$, we have an irreducible canonical curve on the 
canonical model of $S$. In this case, it has already been shown by Reid [19] that $R\left(S, K_{S}\right)$ is generated in degrees $\leq 3$ and related in degrees $\leq 6$ provided that $p_{g} \geq 2$ and $K_{S}^{2} \geq 3$. The main ingredient of his proof is to apply the following fact to that curve:

Lemma 4.8 ([19, p. 246, Theorem]). Let $X$ be an irreducible Gorenstein curve of genus $g \geq 2$, and $E$ a Cartier divisor on $X$ such that $2 E$ is linearly equivalent to $K_{X}$. If $g \geq 4$, then the ring $\bigoplus_{m \geq 0} H^{0}(X, m E)$ is generated in degrees $\leq 3$ and related in degrees $\leq 6$.

We now state our main result in this section, which is (1) of Theorem B:

THEOREM 4.9. Let $S$ be a minimal surface of general type with $q(S)=0, p_{g}(S) \geq 2$ and $K_{S}^{2} \geq 3$. Assume that the variable part of $\left|K_{S}\right|$ contains an irreducible curve and the fixed part $Z$ of $\left|K_{S}\right|$ satisfies $h^{0}\left(Z, K_{Z}\right)=0$. Then the canonical ring is generated in degrees $\leq 3$ and related in degrees $\leq 6$.

Proof. We can assume that the bi-canonical map of $S$ maps $C$ birationally onto the image. In fact, if it is not the case, then $D$ induces on the canonical model of $S$ an irreducible, hyperelliptic canonical curve by Lemma 4.4 and we can apply the above result of Reid.

We may also assume that $Z \neq 0$. Then $M Z$ is a positive even integer. We are going to show that $K_{S}^{2} \geq p_{g}(S)+1$, which is clear when $p_{g}(S)=2$ since $K_{S}^{2} \geq 3$. If $p_{g}(S) \geq 3$, then $M^{2} \geq 2 p_{g}-4$ and

$$
K_{S}^{2}=K_{S} M+K_{S} Z=M^{2}+M Z+K_{S} Z \geq 2 p_{g}(S)-4+M Z+K_{S} Z .
$$

Hence we get $K_{S}^{2} \geq p_{g}(S)+1$. Then it follows from Lemmas 1.4, 4.6 and 4.7 that $R(D, L)$ is generated in degrees $\leq 3$ and related in degrees $\leq 6$. By the hyperplane section principle, $R\left(S, K_{S}\right)$ has the same properties.

We close the section with stating a similar result for irregular surfaces which is obtained analogously as above if we replace $R(D, L)$ by the image $R^{\prime}(D, L)$ of $R\left(S, K_{S}\right) \rightarrow R(D, L)$. Note that the difference between $R^{\prime}(D, L)$ and $R(D, L)$ is only in degrees 1 and 2. Put $V=\operatorname{Im}\left\{H^{0}\left(S, 2 K_{S}\right) \rightarrow\right.$ $\left.\rightarrow H^{0}(D, 2 L)\right\}$. Since $2 K_{S}$ is free, $V$ induces a free linear system on $D$.

THEOREM 4.10. Let $S$ be a minimal irregular surface of general type. Assume that the variable part of $\left|K_{S}\right|$ contains an irreducible curve and the fixed part $Z$ supports at most exceptional sets of rational singular points. 
If $K_{S}^{2} \geq \min \left\{p_{g}(S)+2 q(S), 2 q(S)+4-K_{S} Z\right\}$, then $R\left(S, K_{S}\right)$ is generated in degrees $\leq 4$ and related in degrees $\leq 8$.

Proof. Let $D=C+Z \in\left|K_{S}\right|$ be a general member as above. The exact sequence

$$
0 \rightarrow H^{0}\left(S, K_{S}\right) \rightarrow H^{0}\left(S, 2 K_{S}\right) \rightarrow H^{0}(D, 2 L) \rightarrow H^{1}\left(S, K_{S}\right) \rightarrow 0
$$

gives us $\operatorname{dim} V=K_{S}^{2}+1-q(S)$. Since $Z$ supports at most exceptional sets of rational singular points, we have $H^{0}\left(Z, \mathcal{O}_{Z}(Z)\right)=H^{0}\left(Z, K_{Z}\right)=0$. Hence, as in the proof of Lemma 4.5, we can show that $H^{0}(D, L) \rightarrow H^{0}(C, L)$ is injective and that the Koszul map

$$
\bigwedge^{p} V \otimes H^{0}(D, L) \rightarrow \bigwedge^{p-1} V \otimes H^{0}(D, 3 L)
$$

is injective when $h^{0}(D, L) \leq p$. Then the Koszul map

$$
\bigwedge^{\operatorname{dim} V-2} V \otimes H^{0}(D,(6-m) L) \rightarrow \bigwedge^{\operatorname{dim} V-3} V \otimes H^{0}(D,(8-m) L)
$$

which is clearly injective for $m \geq 6$, is also injective for $m=5$ provided that $h^{0}(D, L) \leq \operatorname{dim} V-2$. Then, by Theorem 1.1, we see that the multiplication map $V \otimes H^{0}(D,(m-2) L) \rightarrow H^{0}(D, m L)$ is surjective for $m \geq 5$ if $h^{0}(D, L) \leq \operatorname{dim} V-2=K_{S}^{2}-q(S)-1$. We have $h^{0}(D, L) \leq p_{g}(S)+q(S)-1 \quad$ and $\quad h^{0}(D, L) \leq h^{0}(C, L) \leq K_{S} C / 2+1 \quad$ by Clifford's theorem. Hence we get $h^{0}(D, L) \leq \operatorname{dim} V-2$ provided that $K_{S}^{2} \geq \min \left\{p_{g}(S)+2 q(S), 2 q(S)+4-K_{S} Z\right\}$. Since we always have $K_{S}^{2} \geq 2 p_{g}(S)$ for irregular surfaces, we get $K_{S}^{2} \geq 2 q(S)+4$ when $\chi\left(\mathcal{O}_{S}\right) \geq 3$.

For $m \geq 9$, the Koszul map

$$
\bigwedge^{\operatorname{dim} V-3} V \otimes H^{0}(D,(8-m) L) \rightarrow \bigwedge^{\operatorname{dim} V-4} V \otimes H^{0}(D,(10-m) L)
$$

is injective, since $h^{0}(D,(8-m) L)=0$. By Theorem 1.1, this shows that

$$
\bigwedge^{2} V \otimes H^{0}(D,(m-4) L) \rightarrow V \otimes H^{0}(D,(m-2) L) \rightarrow H^{0}(D, m L)
$$

is exact at the middle term for $m \geq 9$. In sum, we have shown that the image $R^{\prime}(D, L)$ of the restriction map $R\left(S, K_{S}\right) \rightarrow R(D, L)$ is generated in degrees $\leq 4$ and related in degrees $\leq 8$. Hence we can say the same things for $R\left(S, K_{S}\right)$. 


\section{The case of semi-negative fixed part.}

In this section, we complete the proof of Theorem B by studying the case $h^{1}\left(Z, \mathcal{O}_{Z}\right)>0$ when $Z$ is a numerically disjoint sum of chain connected curves which are semi-negative:

THEOREM 5.1. Let $S$ be a minimal algebraic surface of general type with $p_{g}(S) \geq 2, q(S)=0$ and $K_{S}^{2} \geq 3$. Let $\left|K_{S}\right|=|M|+Z$ be the decomposition into its variable and fixed parts. Suppose that $|M|$ contains an irreducible member and $Z$ does not contain a Francia cycle. If there is a decomposition $Z=\Delta+\Gamma_{1}+\cdots+\Gamma_{n}$ satisfying the following conditions, then $R\left(S, K_{S}\right)$ is generated in degrees $\leq 3$ and related in degrees $\leq 6$ :

(1) $\Delta$ is a curve consisting of $(-2)$-curves (possibly $\Delta=0$ ), $\operatorname{Supp}(\Delta) \cap \operatorname{Supp}(Z-\Delta)=\emptyset$ and $Z-\Delta \neq 0$.

(2) For each $i \in\{1,2, \ldots, n\}, \Gamma_{i}$ is a chain connected curve such that $K_{S} \Gamma_{i}>0, \mathcal{O}_{\Gamma_{i}}\left(-\Gamma_{i}\right)$ is nef, and $\mathcal{O}_{\Gamma_{i}}\left(\Gamma_{j}\right)$ is numerically trivial when $j \neq i$.

The key point is as usual to show the following:

Proposition 5.2. With the same notation and assumptions in Theorem 5.1, $H^{0}\left(S, 4 K_{S}\right)$ is generated by products of elements of lower degrees.

We first give the proof of Theorem 5.1 assuming Proposition 5.2.

Proof of Theorem 5.1. By Lemma 1.4 and Proposition 5.2, it suffices to check that $K_{S}^{2} \geq p_{g}(S)+1+h^{0}\left(Z, K_{Z}\right)$ in view of Lemma 4.6. Let $Z=\Delta+\Gamma_{1}+\cdots+\Gamma_{n}$ be the decomposition as in the statement. We have

$$
h^{0}\left(Z, K_{Z}\right)=\frac{1}{2} Z\left(K_{S}+Z\right)+v, \quad v=h^{0}\left(Z, \mathcal{O}_{Z}\right)
$$

Then the condition $K_{S}^{2} \geq p_{g}(S)+1+h^{0}\left(Z, K_{Z}\right)$ is equivalent to $2 M^{2}+3 M Z \geq 2 p_{g}(S)+2 v+2$. We have $v=h^{0}\left(Z, \mathcal{O}_{Z}\right)=h^{0}\left(\Delta, \mathcal{O}_{4}\right)+$ $+h^{0}\left(Z-\Delta, \mathcal{O}_{Z-4}\right)$ by (1). By an inductive argument using the cohomology long exact sequence for

$$
0 \rightarrow \mathcal{O}_{\Gamma_{i}}\left(-\left(\Gamma_{i+1}+\cdots+\Gamma_{n}\right)\right) \rightarrow \mathcal{O}_{\Gamma_{i}+\cdots+\Gamma_{n}} \rightarrow \mathcal{O}_{\Gamma_{i+1}+\cdots+\Gamma_{n}} \rightarrow 0
$$

one gets $h^{0}\left(Z-\Delta, \mathcal{O}_{Z-\Delta}\right) \leq h^{0}\left(\Gamma_{n}, \mathcal{O}_{\Gamma_{n}}\right)+\sum_{i=1}^{n-1} h^{0}\left(\Gamma_{i},-\left(\Gamma_{i+1}+\cdots+\Gamma_{n}\right)\right)$. Hence $h^{0}\left(Z-\Delta, \mathcal{O}_{Z-\Delta}\right) \leq n$ by (2). We have $h^{0}\left(\Delta, \mathcal{O}_{\Delta}\right)=-\Delta^{2} / 2$, because $h^{1}\left(\Delta, \mathcal{O}_{\Delta}\right)=0$ and $\chi\left(\Delta, \mathcal{O}_{\Delta}\right)=-\Delta\left(K_{S}+\Delta\right) / 2$. It follows from $K_{S} \Delta=$ $=(Z-\Delta) \Delta=0$ that $M \Delta=-\Delta^{2}$. 
By (2), we have $\Gamma_{i}^{2} \leq 0$ and $\Gamma_{i} \Gamma_{j}=0$ for $i \neq j$. Then, Lemma 4.1 shows that $M \Gamma_{i}$ is a positive even integer. Furthermore, we get $M \Gamma_{i} \geq 4$ by Lemma 4.3, since there are no Francia cycles in $Z$ and $K_{S} \Gamma_{i}>0$. It follows $M(Z-\Delta) \geq 4 n$ and we get $M Z \geq 4 n-\Delta^{2}$. Then, since $n \geq v-h^{0}\left(\Delta, \mathcal{O}_{\Delta}\right)=$ $=v+\Delta^{2} / 2$, we get $M Z \geq 2 v+2 n$. Since $M^{2} \geq 2 p_{g}(S)-4$, we get $2 M^{2}+3 M Z \geq 4 p_{g}-8+6 v+6 n$. Hence $2 M^{2}+3 M Z \geq 2 p_{g}(S)+2 v+2$ as wished.

In order to show Proposition 5.2, we slightly change the situation as follows. Let $\sigma: S \rightarrow X$ be the contraction of $\Delta$. Then $X$ is a normal Gorenstein surface with (at most) rational double points and we have $\sigma^{*} \omega_{X}=\mathcal{O}_{S}\left(K_{S}\right), R\left(X, \omega_{X}\right) \simeq R\left(S, K_{S}\right)$. By (1) of Theorem 5.1, we can harmlessly push everything on $S$ down to $X$, which enables us to assume that $\Delta=0$ with the cost that we cannot stay on a non-singular surface. We shall indeed work on such $X$. However, we use the same symbols as before for simplicity: $S$ is now a normal Gorenstein surface with at most rational double points, $Z=\Gamma_{1}+\cdots+\Gamma_{n}$ and $\left|\omega_{S}\right|=|M|+Z$ is the decomposition into the variable and fixed parts. $C \in|M|$ is irreducible, $D=C+Z$ and $L=\omega_{S} \otimes \mathcal{O}_{D}$. Note that $\operatorname{Supp}(Z)$ is entirely contained in the regular locus of $S$. Similarly as in the previous section, it suffices for our purpose to show that $H^{0}(D, 4 L)$ is generated by products of lower degree elements.

We put $W_{m}=\operatorname{Im}\left\{H^{0}(D, m L) \rightarrow H^{0}(C, m L)\right\}$ for a non-negative integer $m$.

LEMMA 5.3. $W_{m}=H^{0}(C, m L)$ when $m \geq 3$, and $\operatorname{dim} W_{2}=h^{0}(C, 2 L)-$ $-h^{0}\left(Z, \mathcal{O}_{Z}\right)+1$.

Proof. For integers $m \geq 2$, consider the exact sequence

$$
H^{0}(D, m L) \rightarrow H^{0}(C, m L) \rightarrow H^{1}(Z, m L-C) \rightarrow H^{1}(D, m L) .
$$

We have $\mathcal{O}_{Z}(m L-C)=\mathcal{O}_{Z}\left(K_{Z}+(m-2) K_{S}\right)$. Since $Z$ is a numerically disjoint sum of chain-connected curves $\Gamma_{i}$ with $K_{S} \Gamma_{i}>0$, we have $H^{1}(Z, m L-C)=0$ for $m \geq 3$ by Lemma 2.2. In fact, by virtue of (2) in Theorem 5.1, one may argue by induction using (5.5) tensored with $\mathcal{O}\left(-(m-2) K_{S}\right)$, in order to get $H^{0}\left(Z,-(m-2) K_{S}\right)=0$. When $m=2$, we have $h^{1}(Z, 2 L-C)=h^{0}\left(Z, \mathcal{O}_{Z}\right)$ by duality, $h^{1}(D, 2 L)=1$ and $h^{1}(C, 2 L)=0$. Hence the assertion follows. 
Similarly as above, using the exact sequence

$$
0 \rightarrow \mathcal{O}_{C}(m L-Z) \rightarrow \mathcal{O}_{D}(m L) \rightarrow \mathcal{O}_{Z}(m L) \rightarrow 0,
$$

we can show that the restriction map $H^{0}(D, m L) \rightarrow H^{0}(Z, m L)$ is surjective for $m \geq 2$. Then, by a diagram chasing, we see that the multiplication map

$$
\begin{gathered}
H^{0}(D, L) \otimes H^{0}(D, 3 L) \\
\bigoplus \\
H^{0}(D, 2 L) \otimes H^{0}(D, 2 L)
\end{gathered} \longrightarrow H^{0}(D, 4 L)
$$

is surjective provided that

$$
H^{0}(Z, 2 L) \otimes H^{0}(Z, 2 L) \rightarrow H^{0}(Z, 4 L)
$$

and

$$
\begin{gathered}
H^{0}(C, L-Z) \otimes H^{0}(C, 3 L) \\
\bigoplus \\
H^{0}(C, 2 L-Z) \otimes W_{2}
\end{gathered} \longrightarrow H^{0}(C, 4 L-Z)
$$

are both surjective. Recall that $\mathcal{O}_{C}(L-Z)=\mathcal{O}_{C}(M)$ and $\mathcal{O}_{C}(2 L-Z)=\omega_{C}$.

LEMMa 5.4. The multiplication map (5.7) is surjective.

Proof. Recall that $\Gamma_{i}$ is chain connected. Since $2 K_{S \mid \Gamma_{i}}-K_{\Gamma_{i}}=$ $=\left(K_{S}-\Gamma_{i}\right)_{\mid \Gamma_{i}}$ which is nef and non-trivial by the assumption, we have $H^{1}\left(\Gamma_{i}, \mathcal{L}\right)=0$ for any invertible sheaf $\mathcal{L}$ on $\Gamma_{i}$ numerically equivalent to $\mathcal{O}_{\Gamma_{i}}\left(2 K_{S}\right)$, by Lemma 2.2 . By the assumption, $\mathcal{O}_{\Gamma_{j}}\left(\Gamma_{i}\right)$ is numerically trivial when $i \neq j$. From the inductive argument using the exact sequences

$$
\begin{aligned}
0 \rightarrow H^{0}\left(\Gamma_{k}, 2 K_{S}-\Gamma_{1}-\cdots-\Gamma_{k-1}\right) & \\
& \rightarrow H^{0}\left(\Gamma_{1}+\cdots+\Gamma_{k}, 2 K_{S}\right) \rightarrow H^{0}\left(\Gamma_{1}+\cdots+\Gamma_{k-1}, 2 K_{S}\right) \rightarrow 0,
\end{aligned}
$$

it follows that the restriction map $H^{0}\left(Z, 2 K_{S}\right) \rightarrow H^{0}\left(\Gamma_{i}, 2 K_{S}\right)$ is surjective. At the same time, we see that, in order to show that (5.7) is surjective, it suffices to show that $H^{0}\left(\Gamma_{i}, L_{1}\right) \otimes H^{0}\left(\Gamma_{i}, 2 K_{S}\right) \rightarrow H^{0}\left(\Gamma_{i}, L_{1}+2 K_{S}\right)$ is surjective for each $\Gamma_{i} \preceq Z$ and for any line bundle $L_{1}$ on $\Gamma_{i}$ which is numerically equivalent to $2 K_{S}$ on $\Gamma_{i}$. But it is nothing but a special case of Theorem 2.6.

We next consider the map (5.8). We let $\zeta$ denote the fixed part of $\left|M_{\mid C}\right|$, that is, the smallest effective Cartier divisor $\zeta$ on $C$ such that $\left|\mathcal{O}_{C}(M-\zeta)\right|$ is free from base points. We put $N=M_{\mid C}-\zeta$. 
LEMMA 5.5. The image of the multiplication map

$$
H^{0}(C, M) \otimes H^{0}(C, 3 L) \rightarrow H^{0}(C, 4 L-Z)
$$

is the subspace spanned by elements vanishing on $\zeta$.

Proof. The kernel of the restriction map $H^{0}(C, 4 L-Z) \rightarrow$ $\rightarrow H^{0}(\zeta, 4 L-Z)$ is isomorphic to $H^{0}(C, 4 L-Z-\zeta)$, and we have an injection $H^{0}(C, N) \simeq H^{0}(C, M-\zeta) \rightarrow H^{0}(C, M)$ by multiplying the section defining $\zeta$. Hence it suffices to show that the multiplication map

$$
H^{0}(C, N) \otimes H^{0}(C, 3 L) \rightarrow H^{0}(C, 4 L-Z-\zeta)
$$

is surjective. We have $\mathrm{Bs}|N|=\emptyset$. By Castelnuovo's lemma, it suffices to show that $H^{1}(C, 3 L-N)=0$. Since $\mathcal{O}_{C}(3 L-N) \simeq \mathcal{O}_{C}\left(K_{C}+2 Z+\zeta\right)$, we in fact have $h^{1}(C, 3 L-N)=0$.

The following completes the proof of Proposition 5.2.

LEMma 5.6. The multiplication map (5.8) is surjective.

Proof. By the previous lemma, it is sufficient to show that the composite of the multiplication map $H^{0}\left(C, K_{C}\right) \otimes W_{2} \rightarrow H^{0}(C, 4 L-Z)$ and the restriction map $H^{0}(C, 4 L-Z) \rightarrow H^{0}(\zeta, 4 L-Z)$ is surjective. We claim that the restriction map $H^{0}\left(C, K_{C}\right) \rightarrow H^{0}\left(\zeta, K_{C}\right)$ is surjective. This can be seen as follows. Note that we have $h^{0}\left(C, \mathcal{O}_{C}(\zeta)\right)=1$, because $\zeta$ cannot move. Then it follows from the Riemann-Roch theorem that $h^{1}(C, \zeta)=h^{0}\left(C, K_{C}-\zeta\right)=$ $=p_{a}(C)-\operatorname{deg} \zeta$. By the dimension count, this implies that $H^{0}\left(C, K_{C}\right) \rightarrow$ $\rightarrow H^{0}\left(\zeta, K_{C}\right)$ is surjective. Then, since $\left|2 K_{S}\right|$ is free from base points, the multiplication map $H^{0}\left(\zeta, K_{C}\right) \otimes W_{2 \mid \zeta} \rightarrow H^{0}(\zeta, 4 L-Z)$ is clearly surjective. This show the claim and we see that (5.8) is surjective.

\section{Appendix.}

In this appendix, we state some results about canonical algebras of curves on a smooth surface in order to supplement [13].

Let $D$ be a numerically 1-connected curve on a smooth surface such that $p_{a}(D) \geq 2$ and $K_{D}$ is nef. In [13], we studied the canonical ring $R\left(D, K_{D}\right)=$

$=\bigoplus_{m \geq 0} H^{0}\left(D, m K_{D}\right)$ and showed that it is generated in degrees $\leq 3$.

THeOREm 6.1. Let D be a numerically 1-connected curve on a smooth surface such that $p_{a}(D) \geq 2$ and $K_{D}$ is nef. Then the canonical ring of $D$ is generated in degrees $\leq 3$ and related in degrees $\leq 6$. 
Proof. Let the notation be as in Lemma 1.4. As we remarked above, we have $d \leq 3$ by [13, Theorem II]. By [13, Corollary 1.2.3], we have $d_{0}=4$ and $d_{1}=6$. Now, apply Lemma 1.4 .

Via Nakayama's lemma, this also applies to the relative canonical algebra of a relatively minimal fibration $f: S \rightarrow B$ over a non-singular curve $B$ whose general fibre is of genus $\geq 2$. Recall that we have [13, Theore$\mathrm{m}$ III] for generators.

THEOREM 6.2. The relative canonical algebra for a relatively minimal fibration of curves of genus $\geq 2$ is generated in degrees $\leq 4$ and related in degrees $\leq 8$. If furthermore there are no multiple fibres whose canonical system has a (-1)-elliptic cycle as a fixed component, then the relative canonical algebra is generated in degrees $\leq 3$ and related in degrees $\leq 6$.

A further application goes over the relative canonical algebra for a normal surface singularity. Let $(V, p)$ be a normal surface singularity and $\pi: S \rightarrow V$ the minimal resolution. We regard $S$ as a sufficiently small, strongly pseudo-convex neighbourhood of $A=\pi^{-1}(p)$. Let $D$ be the fundamental cycle on $A$. We are interested in the canonical ring $R\left(S, K_{S}\right)=\bigoplus_{m=0}^{\infty} H^{0}\left(S, m K_{S}\right)$. Laufer [15] showed that it is generated in degrees $\leq 3$. The following can be found in [14] and [15]:

THEOREM 6.3. Let $D$ be the fundamental cycle on $A$ and $n$ a positive integer. Then $H^{1}(n D, L)=0$ for any line bundle $L$ on $S$ such that $L-K_{S}$ is nef on D. Furthermore, the ring $R\left(n D, K_{S}\right)=\bigoplus_{m=0}^{\infty} H^{0}\left(n D, m K_{S}\right)$ is generated in degrees $\leq 3$.

Let $n$ be a positive integer. We claim that the Koszul sequence

$$
\begin{aligned}
\bigwedge^{2} H^{0}\left(n D, 2 K_{S}\right) \otimes & H^{0}\left(n D,(m-4) K_{S}\right) \\
& \rightarrow H^{0}\left(n D, 2 K_{S}\right) \otimes H^{0}\left(n D,(m-2) K_{S}\right) \rightarrow H^{0}\left(n D, m K_{S}\right)
\end{aligned}
$$

is exact at the middle term for $m \geq 7$. By Theorem 1.1, it suffices to show that

$$
\begin{aligned}
\bigwedge_{r_{n}-2} H^{0}\left(n D, 2 K_{S}\right) \otimes H^{0}(n D & \left., K_{n D}-(m-6) K_{S}\right) \\
& \rightarrow \bigwedge^{r_{n}-3} H^{0}\left(n D, 2 K_{S}\right) \otimes H^{0}\left(n D, K_{n D}-(m-8) K_{S}\right)
\end{aligned}
$$


is injective for $m \geq 7$, where $r_{n}=h^{0}\left(n D, 2 K_{S}\right)-1$. By Serre duality, we have $H^{0}\left(n D, K_{n D}-(m-6) K_{S}\right)^{\vee} \simeq H^{1}\left(n D,(m-6) K_{S}\right)$, which vanishes for any integer $m$ with $m \geq 7$ by the above theorem. Hence we conclude that $R\left(n D, K_{S}\right)$ is related in degrees $\leq 6$.

Note that, for any positive cycle $\Delta$ supported on $A$, we can find a positive integer $n$ such that $\Delta \preceq n D$. We have $\pi_{*} \mathcal{F}^{\wedge}=\lim _{\Delta} H^{0}\left(\pi^{-1}(p), \mathcal{F} \otimes \mathcal{O}_{\Delta}\right)$ for a coherent sheaf $\mathcal{F}$ on $S$. Therefore, we get the following as conjectured by Reid:

THEOREM 6.4. The relative canonical algebra for a normal surface singularity is generated in degrees $\leq 3$ and related in degrees $\leq 6$.

\section{REFERENCES}

[1] F. Catanese, Surfaces with $K^{2}=p_{g}=1$ and their period mapping, Algebraic geometry (Proc. Summer Meeting, Univ. Copenhagen, Copenhagen, 1978), pp. 1-29, Lecture Notes in Math., 732, Springer, Berlin, 1979.

[2] F. Catanese, On a class of surfaces of general type, Algebraic Surfaces, CIME 1977, Liguori (1981), pp. 269-284.

[3] F. Catanese - C. Ciliberto, Surfaces with $p_{g}=q=1$, Problems in the theory of surfaces and their classification (Cortona, 1988), pp. 49-79, Sympos. Math., XXXII, Academic Press, London, 1991.

[4] C. Ciliberto, Sul grado dei generatori dell'anello canonico di una superficie di tipo generale, Rend. Sem. Mat. Univ. Politec. Torino, 41:3 (1983/84), pp. 83111.

[5] C. Ciliberto - P. Francia - M. Mendes Lopes, Remarks on the bicanonical map for surfaces of general type, Math. Z., 224 (1997), pp. 137-166.

[6] O. Debarre, Inégalités numériques pour les surfaces de type général, Bull. Soc. Math. France, 110 (1982), pp. 319-346.

[7] P. Francia, On the base points of the bicanonical system, Problems in the theory of surfaces and their classification (Cortona, 1988), pp. 141-150, Sympos. Math., XXXII, Academic Press, London, 1991.

[8] M. Green, The canonical ring of a variety of general type, Duke Math. J., 49 (1982), pp. 1087-1113.

[9] M. GReEn, Koszul cohomology and geometry of projective varieties, J. Diff. Geom., 19 (1984), pp. 125-171.

[10] E. HoRIKawa, Algebraic surfaces of general type with small $c_{1}^{2}$. I, Ann. of Math., 104 (1976), pp. 357-387.

[11] E. HonIKaWA, Algebraic surfaces of general type with small $c_{1}^{2}$. II, Invent. Math., 37 (1976), pp. 121-155.

[12] E. HorikaWA, Algebraic surfaces of general type with small $c_{1}^{2}$. V, J. Fac. Sci. Univ. Tokyo Sect. IA Math., 28 (1981), pp. 745-755.

[13] K. Konno, 1-2-3 theorem for curves on algebraic surface, J. reine. angew. Math., 533 (2001), pp. 171-205. 
[14] H. B. LAUFER, Weak simultaneous resolution for deformations of Gorenstein surface singularities, Proc. Symp. Pure Math., 40 (1983), pp. 1-29.

[15] H. B. LAUFER, Generation of 4-pluricanonical forms for surface singularities, Amer. J. Math., 109 (1987), pp. 571-589.

[16] M. Mendes Lopes, Adjoint systems on surfaces, Bollettino U.M.I. (7), 10-A (1996), pp. 169-179.

[17] M. Mendes Lopes, The degree of the generators of the canonical ring of surfaces of general type with $p_{g}=0$, Arch. Math., 69, no. 5 (1997), pp. 435-440.

[18] M. Nagata, On rational surfaces I, Mem. Coll. Sci. Kyoto Univ. Ser. A, 32 (1960), pp. 351-370.

[19] M. REID, Infinitesimal view of extending a hyperplane section - deformation theory and computer algebra, Lect. Notes in Math., 1417, Algebraic Geometry Proceedings, L’Aquila 1988, A. J. Sommese et al., eds. 1990, Springer-Verlag, pp. 214-286.

[20] M. REID, Chapters on Algebraic Surfaces, Complex algebraic geometry (Park City, UT, 1993), pp. 3-159, IAS/Park City Math. Ser., 3, Amer. Math. Soc., Providence, RI, 1997.

[21] M. REID, 1-2-3, unpublished note.

[22] I. REIDER, Vector bundles of rank 2 and linear systems on algebraic surfaces, Ann. of Math., 127 (1988), pp. 309-316.

[23] G. XIAo, Surfaces fibrées en courbes de genre deux, Lecture Notes in Mathematics, 1137, Springer-Verlag, Berlin, 1985.

[24] G. Xiao, Degree of the bicanonical map of a surface of general type, Amer. J. Math., 112, no. 5 (1990), pp. 713-736.

Manoscritto pervenuto in redazione il 21 maggio 2007. 
